\title{
Gravitino dark matter, nonthermal leptogenesis, and low reheating temperature in no-scale Higgs inflation
}

\author{
Waqas Ahmed®, ${ }^{1, *}$ Athanasios Karozas $\odot,{ }^{2, \dagger}$ and George K. Leontaris $\oplus^{2, \dagger}$ \\ ${ }^{1}$ School of Mathematics and Physics, Hubei Polytechnic University, \\ No. 16 North Road, Guilin, Huangshi, 435003 Hubei, China \\ ${ }^{2}$ Physics Department, Theory Division, University of Ioannina, GR-45110 Ioannina, Greece
}

(Received 14 April 2021; accepted 17 August 2021; published 21 September 2021)

\begin{abstract}
We revisit Higgs inflation in the framework of a minimal extension of the Standard Model gauge symmetry by a $U(1)_{B-L}$ factor. Various aspects are taken into account, with particular focus on the role of the supersymmetry-breaking (SUSY) scale and the cosmological constraints associated with the gravitino. The scalar potential of the model is considered in the context of no-scale supergravity consisting of the $F$ part constructed from the Kähler function, the $D$ terms, and soft SUSY contributions. We investigate several limiting cases and by varying the SUSY scale from a few TeV up to intermediate energies, for a spectral index around $n_{s} \sim 0.9655$ and reheating temperature $T_{r} \leq 10^{9} \mathrm{GeV}$, we find that the value of the tensor-to-scalar ratio ranges from $r \approx 10^{-3}$ to $10^{-2}$. Furthermore, it is shown that for certain regions of the parameter space the gravitino can live sufficiently long, and as such is a potential candidate for a dark matter component. In general, the inflationary scenario is naturally implemented and it is consistent with nonthermal leptogenesis, whereas the dominant decay channel of the inflaton yields right-handed neutrinos. Other aspects of cosmology and particle physics phenomenology are briefly discussed. Finally, we investigate the case where the inflaton is initially relaxed in a false minimum and estimate its probability to decay to the true vacuum.
\end{abstract}

DOI: $10.1103 /$ PhysRevD.104.055025

\section{INTRODUCTION}

The theory of cosmological inflation has indisputable advantages. Among other implications, it provides convincing answers to the flatness and horizon problems [1-3], and explains the origin of the large-scale structure of the Universe.

During the last few decades numerous scenarios have been proposed to formulate a detailed microscopic mechanism for a complete theory of inflation with successful predictions for all related observables. The cosmic microwave background (CMB) observations, however, put rather tight constraints on many inflationary theories [4,5]. Several models such as those with quartic and quadratic potentials fail to satisfy the bound on the tensor-to-scalar ratio (which, according to Planck 2018 results, is $r<0.05$ at the $95 \%$ confidence level [5]), and hence such models are

\footnotetext{
*waqasmit@nankai.edu.cn, waqasmit@hbpu.edu.cn †akarozas@uoi.gr

"leonta@uoi.gr
}

Published by the American Physical Society under the terms of the Creative Commons Attribution 4.0 International license. Further distribution of this work must maintain attribution to the author(s) and the published article's title, journal citation, and DOI. Funded by SCOAP. ruled out. On the other side, one of the most successful inflationary models, which is in accordance with the Planck 2018 results, is the Starobinsky model [1] which predicts $n_{s}=1-\frac{2}{N_{0}}$ and $r=\frac{12}{N_{0}^{2}} \sim(0.002-0.004)$, where $N_{0}$ represents the numbers of $e$-folds. The Starobinsky inflationary potential has been studied extensively in the literature within various contexts, where it was shown that this model can be derived in the context of no-scale supergravity [6-13]. The simplest version of the Starobinsky proposal is equivalent to an inflationary model in which the scalar field couples nonminimally to gravity. A natural choice for the inflaton field in grand unified theories (GUTs) in the context of supergravity is the Higgs field breaking the GUT symmetry [14-18]. Inflation with a Standard Model (SM)-like Higgs boson in the minimal supersymmetric Standard Model (MSSM) has also been proposed $[10,12,19]$. Also, in Refs. [20,21] nonsupersymmetric models with nonminimal Higgs inflation were discussed. Several other ideas including chaotic, hybrid, and hilltop inflation have been proposed and investigated in detail [22]. Some of these scenarios can also be realized in a string theory framework where the role of the inflaton field is played by some modulus.

In particular, hybrid inflation is one of the most promising models of inflation, and can be naturally realized within the context of supergravity theories. This scenario is 
based on the inclusion of two scalar fields [23], with the first one realizing the slow-roll inflation and the second one, dubbed the "waterfall" field, triggering the end of the inflationary epoch. While in the standard hybrid inflationary scenario [24-26] in the supersymmetric GUTs the symmetry is broken at the end of inflation, in the case of shifted [27] and smooth inflation [28] the symmetry breaking occurs during inflation, and thus magnetic monopoles and other topological defects are inflated away.

On the side of particle physics, the experimental data collected at the Large Hadron Collider (LHC) has put strong constraints on the conventional SUSY scenarios with superpartner masses around the electroweak scale. The so-far null SUSY experimental results have triggered significant interest in alternative SUSY-scale scenarios, such as high-scale SUSY and split SUSY, among others $[29,30]$. Taking into account the possible connection between inflationary dynamics and SUSY-scale phenomenology, it will be interesting to study the effects among various SUSY-scale scenarios with a successful model of inflation.

In this paper we study inflation in a supergravity context where a no-scale Kähler potential is assumed. We consider the framework of the SM gauge symmetry augmented by a $U(1)_{B-L}$ factor [31-33]. In this background, after employing the mechanism proposed in Refs. [34,35] for dynamically generating a $\mu$ term, we investigate the implementation of cosmic inflation and its interplay with the issues of SUSY like scale, nonthermal leptogenesis and gravitino dark matter. We show that for TeV and split SUSY scenarios the tensor-to-scalar ratio remains as low as $r \approx \mathcal{O}\left(10^{-3}\right)$, while for a high-scale SUSY scenario $r$ takes higher values of the order $r \sim \mathcal{O}\left(10^{-2}\right)$ with the spectral index fixed at its central value $n_{s}=0.9655$ and the reheating temperature $T_{r}<5 \times 10^{9} \mathrm{GeV}$. We also discuss the process of nonthermal leptogenesis and show that the dominant inflaton decay channel yields right-handed neutrinos. For gravitino cosmology, we explore three possibilities: stable, unstable long-lived, and unstable short-lived gravitinos. For the various cases we investigate the reheating and cosmological gravitino constraints, and we find a consistent inflationary scenario that gives rather concrete predictions regarding supersymmetric dark matter and LHC phenomenology. We complete our analysis with an investigation of certain regions of the parameters that yield a potential containing a false minimum. We examine possible scenarios of quantum tunneling effects and compute the decay probability of such vacua to the true vacuum.

The layout of the paper is as follows. In Sec. II we describe the basic features of the model including the superfields, their charge assignments, and the superpotential constrained by a $U(1) R$ symmetry. The inflationary setup is described in Sec. III. The numerical analysis is presented in Sec. IV, containing the prospects of observing primordial gravity waves and nonthermal leptogenesis.
In Secs. V and VI we discuss the gravitino cosmology and quantum tunneling. Our conclusions are summarized in Sec. VII.

\section{DESCRIPTION OF THE MODEL}

In this section we present the basic features regarding the gauge symmetry and the spectrum of the effective model in which the inflationary scenario will be implemented. The gauge symmetry is the Standard Model gauge group extended by a $U_{B-L}$ Abelian gauge symmetry,

$G_{B-L}=S U(3)_{C} \times S U(2)_{L} \times U(1)_{Y} \times U(1)_{B-L}$.

The particle content of the model contains the MSSM matter and Higgs representations, three singlets accommodating the right-handed neutrinos $N_{i}^{c}$, a neutral scalar singlet $S$, and a pair of Higgs singlets $H, \bar{H}$. These are listed in Table I, where in addition to the transformation properties under Eq. (2.1) the "charges" under the global symmetries $R, B, L$ also are shown.

When the $H, \bar{H}$ singlet Higgs fields acquire vacuum expectation values (VEVs), they break the $U(1)_{B-L}$ symmetry and, at the same time, they provide Majorana masses to the right-handed neutrinos. For the field content and the corresponding charge assignments given in Table I, the renormalizable superpotential of the model is

$$
\begin{aligned}
W= & y_{u} H_{u} q u^{c}+y_{d} H_{d} q d^{c}+y_{e} H_{d} L e^{c}+y_{\nu} H_{u} L N^{c} \\
& +\kappa S\left(\bar{H} H-M^{2}\right)+\lambda S H_{u} H_{d}+\beta H N^{c} N^{c} .
\end{aligned}
$$

The first line contains the Yukawa sector providing Dirac masses to the up and down quarks, charged leptons, and

TABLE I. Superfield content of the model, the corresponding representations under the local gauge symmetry $U_{B-L}$, and the properties with respect to the extra global symmetries.

\begin{tabular}{lcccr}
\hline \hline SUPERFIELDS & $\begin{array}{c}\text { REPRESENTATIONS } \\
\text { UNDER } G_{B-L}\end{array}$ & \multicolumn{3}{c}{$\begin{array}{c}\text { GLOBAL } \\
\text { SYMMETRIES }\end{array}$} \\
\hline & & $R$ & $B$ & $L$ \\
\hline \multicolumn{1}{c}{ MATTER FIELDS } & & & \\
$e_{i}^{c}$ & $(\mathbf{1}, \mathbf{1}, 1,1)$ & 1 & 0 & -1 \\
$N_{i}^{c}$ & $(\mathbf{1}, \mathbf{1}, 0,1)$ & 1 & 0 & -1 \\
$L_{i}$ & $(\mathbf{1}, \mathbf{2},-1 / 2,-1)$ & 1 & 0 & 1 \\
$u_{i}^{c}$ & $(\mathbf{3}, \mathbf{1},-2 / 3,-1 / 3)$ & 1 & $-1 / 3$ & 0 \\
$d_{i}^{c}$ & $(\mathbf{3}, \mathbf{1}, 1 / 3,-1 / 3)$ & 1 & $-1 / 3$ & 0 \\
$Q_{i}$ & $(\overline{\mathbf{3}}, \mathbf{2}, 1 / 6,1 / 3)$ & 1 & $1 / 3$ & 0 \\
& $(\mathbf{1}, \mathbf{2},-1 / 2,0)$ & 0 & 0 & 0 \\
$H_{d}$ & $(\mathbf{1}, \mathbf{2}, 1 / 2,0)$ & 0 & 0 & 0 \\
$H_{u}$ & $(\mathbf{1}, \mathbf{1}, 0,0)$ & 2 & 0 & 0 \\
$S$ & $(\mathbf{1}, \mathbf{1}, 0,2)$ & 0 & 0 & -2 \\
$\bar{H}$ & $(\mathbf{1}, \mathbf{1}, 0,-2)$ & 0 & 0 & 2 \\
$H$ & & & & \\
\hline \hline
\end{tabular}


neutrinos. The corresponding Yukawa couplings are denoted by $y_{u}, y_{d}, y_{e}$, and $y_{\nu}$, and the family indices are generally suppressed for simplicity. The tree-level terms in the second line involve the extra fields beyond the MSSM spectrum. The first term describes the standard supersymmetric hybrid inflation model with $M$ being a GUTscale mass parameter and $\kappa$ a coupling constant coefficient. The second term dynamically generates a $\mu$ term for the model when the singlet scalar $S$ receives a nonzero VEV. The $H$ and $\bar{H}$ fields carry nonzero $B-L$ charges and their VEVs spontaneously break the $U(1)_{B-L}$ symmetry. Furthermore, $H$ is responsible for generating a Majorana mass for the right-handed neutrinos through the last term in Eq. (2.2). By virtue of the extra global symmetries, the model is protected from dangerous proton decay operators and $R$-parity-violating terms. For the same reason, bilinear terms of the forms $H_{u} H_{d}$ and $H \bar{H}$ are absent too. Following this short description of the salient features of the model, in the next section we proceed with the inflationary dynamics.

\section{INFLATION POTENTIAL}

We compute the effective scalar potential considering contributions from the F and D sectors, as well as soft supersymmetry-breaking terms. The superpotential terms relevant for inflation are

$$
W \supset \kappa S\left(\bar{H} H-M^{2}\right)+\lambda S H_{u} H_{d}+\beta H N^{c} N^{c} .
$$

We consider a no-scale structure Kähler potential which, after including contributions of the relevant fields in the present model, takes the form (from now on we set the reduced Planck mass to unity, $M_{\mathrm{Pl}}=1$ )

$$
\begin{aligned}
K= & -3 \log \left[T+T^{*}\right. \\
& -\frac{1}{3}\left(H H^{*}+\bar{H} \bar{H}^{*}+H_{u} H_{u}^{*}+H_{d} H_{d}^{*}+S^{\dagger} S+N^{c \dagger} N^{c}\right) \\
& \left.+\frac{\xi}{3}\left(H \bar{H}+H^{*} \bar{H}^{*}\right)+\frac{\zeta}{3}\left(H_{u} H_{d}+H_{u}^{*} H_{d}^{*}\right)\right]
\end{aligned}
$$

where $T, T^{*}$ are Kähler moduli fields and $\xi, \zeta$ are dimensionless parameters. For later convenience, we define

$$
\begin{aligned}
\Delta= & {\left[T+T^{*}\right.} \\
& -\frac{1}{3}\left(H H^{*}+\bar{H} \bar{H}^{*}+H_{u} H_{u}^{*}+H_{d} H_{d}^{*}+S^{\dagger} S+N^{c \dagger} N^{c}\right) \\
& \left.+\frac{\xi}{3}\left(H \bar{H}+H^{*} \bar{H}^{*}\right)+\frac{\zeta}{3}\left(H_{u} H_{d}+H_{u}^{*} H_{d}^{*}\right)\right]
\end{aligned}
$$

and hence Eq. (3.2) is simply written as $\mathrm{K}=-3 \log \Delta$. Furthermore, we introduce the standard definition of the Kähler function

$$
G=K+\log |W|^{2} \equiv K+\log W+\log W^{*} .
$$

Then, the F-term potential is given by

$$
V_{F}=e^{G}\left(G_{i} G_{i j^{*}}^{-1} G_{j^{*}}-3\right),
$$

where $G_{i}\left(G_{j^{*}}\right)$ are the derivatives with respect to the various scalar fields appearing in Eq. (3.2). Using the above ingredients, the F-term potential (3.4) takes the form

$$
\begin{aligned}
V_{F}= & \frac{1}{\Delta^{2}}\left[\kappa^{2}\left(M^{2}-H \bar{H}\right)^{2}+\kappa^{2} S^{2}\left(H^{2}+\bar{H}^{2}\right)\right. \\
& +\lambda^{2}\left(H_{u}^{2} H_{d}^{2}+S^{2} H_{u}^{2}+S^{2} H_{d}^{2}\right) \\
& +N^{c 2}\left(\beta^{2} N^{c 2}+4 \beta^{2} \bar{H}^{2}+2 \beta \kappa S H\right) \\
& \left.-2 \kappa \lambda H \bar{H} H_{u} H_{d}-2 \kappa \lambda M^{2} H_{u} H_{d}\right] .
\end{aligned}
$$

When the Higgs doublets $H_{u}, H_{d}$, and the various singlet fields $H, \bar{H}, S, N^{c}$ are eliminated and only the Kähler moduli are present, the no-scale structure of the Kähler potential implies that the effective F-term potential vanishes identically, $V_{F} \equiv 0$. We first explore inflation along the $H$ direction, starting with the supersymmetric global minima of the potential. It can be observed that the global minimum of the above potential lies at

$$
S^{0}=H_{u}^{0}=H_{d}^{0}=N^{c 0}=0
$$

and

$$
H^{0}=0, \quad H^{0}= \pm M
$$

where $H^{0}=0$ corresponds to an extremum and $H^{0}= \pm M$ to local minima.

Next, we turn to the D-term potential. For the fields carrying $B-L$ quantum numbers (shown in Table I and denoted collectively here as $\phi_{i}$ ), the D-term potential is

$$
V_{D}=\frac{1}{2} D_{a}^{p} D_{a}^{p}
$$

where

$$
D_{a}^{p}=-g_{a} K_{, \phi_{i}}\left[t_{a}^{p}\right]_{i}^{j} \phi_{j}
$$

for $S U(N)$ groups and $D^{a}$ is defined as

$$
D_{a}^{p}=-g_{a} K_{, \phi_{i}}\left[t_{a}^{p}\right]_{i}^{j} \phi_{j}-g_{a} q_{i} \varsigma
$$

in the presence of $U(1)$ symmetries. Here $q_{i}$ is the charge under $U(1)$, the symbol $\varsigma$ stands for the FayetIliopoulos coupling constant, and $K_{, \phi_{i}} \equiv d K / d \phi_{i}$. The $t_{a}^{p}$ are the generators of the corresponding group $G$ and $p=1, \ldots, \operatorname{dim}(G)$. The D-term potential can be written as 


$$
\begin{aligned}
V_{D}= & \frac{g_{b}^{2}}{2 \Delta^{2}}\left[\phi_{i}^{*}\left(t_{b}^{p}\right)_{i}^{j} \phi_{j}-\zeta \bar{\phi}\left(t_{b}^{p}\right)_{i}^{j} \phi_{j}\right]^{2} \\
& +\frac{g_{B-L}^{2}}{2 \Delta^{2}}\left[\phi_{i}^{*}\left(t_{B-L}^{p}\right)_{i}^{j} \phi_{j}-\xi \bar{\phi}\left(t_{B-L}^{p}\right)_{i}^{j} \phi_{j}-q_{i} \zeta\right]^{2},
\end{aligned}
$$

where $g_{b}(b=1,2,3)$ and $g_{B-L}$ correspond to the $S U(3)_{c}$, $S U(2)_{L}, U(1)_{Y}$, and $U(1)_{B-L}$ gauge couplings, respectively. For our purposes, it suffices to work along $D$-flat directions where the $D$-term potential $V_{D}$ vanishes. Restricting to the scalar fields, we first observe that they transform trivially under $S U(3)_{c}$, and thus the corresponding $D$ term is zero. The other three contributions are

$$
\begin{aligned}
V_{D}= & \frac{g_{1}^{2}}{2 \Delta^{2}}\left[\frac{1}{2}\left|H_{u}\right|^{2}-\frac{1}{2}\left|H_{d}\right|^{2}-\zeta\left(\frac{1}{2} \bar{H}_{d} H_{u}-\frac{1}{2} \bar{H}_{u} H_{d}\right)\right] \\
& +\frac{g_{2}^{8}}{2 \Delta^{2}}\left[H_{u}^{*} \sigma^{p} H_{u}+H_{d}^{*} \sigma^{p} H_{d}-\zeta\left(\bar{H}_{d} \sigma^{p} H_{u}+\bar{H}_{u} \sigma^{p} H_{d}\right)\right]^{2} \\
& +\frac{g_{B-L}^{2}}{2 \Delta^{2}}\left[2|\bar{H}|^{2}-2|H|^{2}-\xi(2 H \bar{H}-2 \bar{H} H)+\left(q_{H}+q_{\bar{H}}\right) \zeta\right]^{2} .
\end{aligned}
$$

Here $\sigma^{p}, p=1,2,3$ are the $S U(2)_{L}$ generators (Pauli matrices) and $H_{u}, H_{d}$ are the MSSM doublet Higgs fields, which in component notation will be written as

$$
H_{u}=\left(\begin{array}{c}
H_{u}^{+} \\
H_{u}^{0}
\end{array}\right), \quad H_{d}=\left(\begin{array}{c}
H_{d}^{0} \\
H_{d}^{-}
\end{array}\right) .
$$

Using the $S U(2)_{L}$ transformation, it is possible to rotate the Higgs boson fields into the neutral directions $H_{u}^{0}$ and $H_{d}^{0}$.

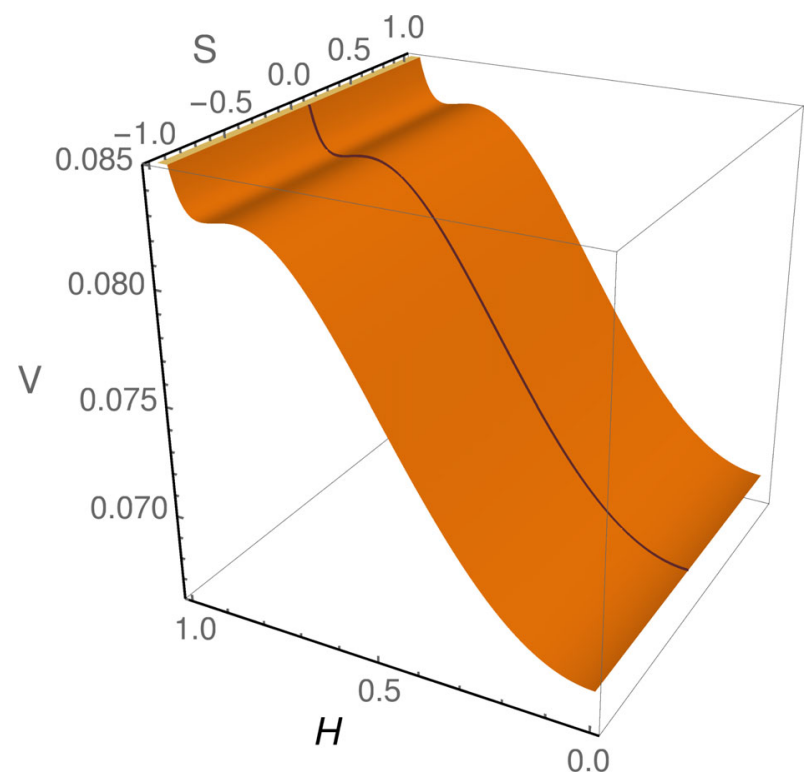

A $D$-flat direction can be achieved with $H_{u}^{0}=H_{d}^{0}$ and $\bar{H}=H$ [36]. Finally, we may also include contributions from explicit soft SUSY-breaking terms of the form

$$
\begin{aligned}
V_{\text {soft }}= & m^{2}\left|\phi_{i}\right|^{2}+\left(\kappa A_{\kappa} S H \bar{H}+\lambda A_{\mu} S H_{u} H_{d}+\beta A_{N^{c}} \bar{H} N^{c} N^{c}\right. \\
& \left.-a_{s} S \kappa M^{2}+\text { H.c. }\right),
\end{aligned}
$$

where $A_{\kappa}=A_{\mu}=A_{N^{c}}$ are the complex coefficients of the trilinear soft SUSY-breaking terms. Along the inflationary trajectory $S$ is zero, so the corresponding trilinear terms do not contribute. Then, for $S=0$ the total effective potential $V(\varphi)=V_{F}+V_{D}+V_{\text {soft }}$ is

$$
V(\varphi)=\frac{9 \kappa^{2}\left(M^{2}-H^{2}\right)^{2}}{\left(3\left(T+T^{*}\right)-2(1-\xi) H^{2}\right)^{2}}+2 m^{2} H^{2} .
$$

The shape of the effective potential is presented in Fig. 1. The left panel represents the potential as a function of $H$ and $S$ with the black curve corresponding to the $S=0$ direction. The right panel shows the potential as a function of $H$ for various values of $\xi$ and $S=0$. Along the inflationary trajectory the vacuum energy density is nonzero. The nonzero field value during inflation also breaks $U(1)_{B-L}$ and the symmetry reduces to the SM. Since the gauge symmetry is broken during inflation, monopoles and cosmic strings are inflated away.

We define the inflaton field as $\varphi=2 H$ and the modulus complex fields as $T=(u+i v)$, and hence $T+T^{*}=2 u$. Then, the potential along the inflationary track is

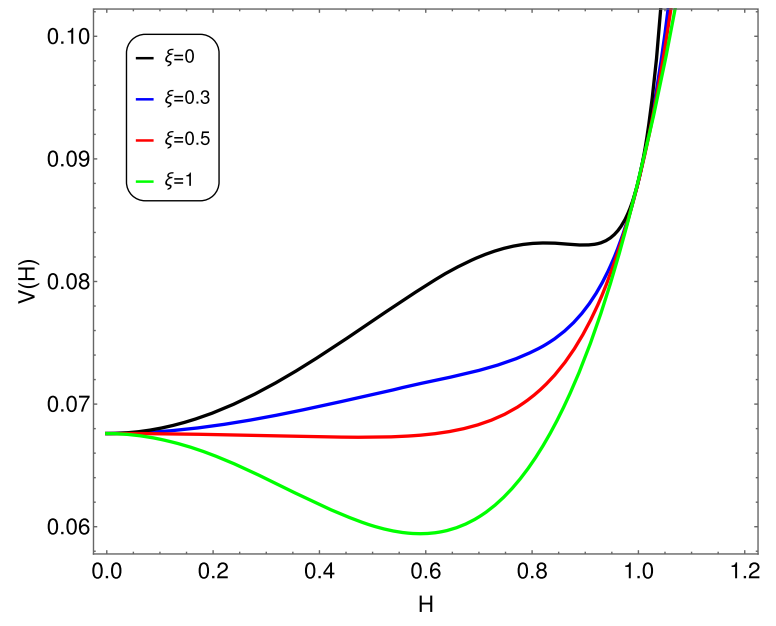

FIG. 1. In the left panel we show the shape of the potential in three dimensions as a function of $S$ and $H$. In the right panel we show the shape of the potential along the $H$ direction for different values of $\xi$. In both plots we have fixed the other parameters as $M=1, m=0.2$, $\kappa=0.26$, and $T=T^{*}=\frac{1}{2}$. 


$$
\begin{aligned}
V(\varphi) & =\frac{\kappa^{2}\left(M^{2}-\frac{\varphi^{2}}{4}\right)^{2}}{\left(2 u-\left(\frac{1-\xi}{6}\right) \varphi^{2}\right)^{2}}+\frac{m^{2}}{2} \varphi^{2} \\
& =\frac{V_{0}\left(1-\frac{\varphi^{2}}{4 M^{2}}\right)^{2}}{\left(2 u-\left(\frac{1-\xi}{6}\right) \varphi^{2}\right)^{2}}+\frac{m^{2}}{2} \varphi^{2},
\end{aligned}
$$

where $V_{0}=\kappa^{2} M^{4}$. However, the inflaton field $\varphi$ is not canonically normalized since its kinetic energy terms take the form

$$
\mathcal{L}(\varphi)=\frac{2 u-\frac{\varphi^{2}}{6} \xi(1-\xi)}{2\left(2 u-\frac{1}{6}(1-\xi) \varphi^{2}\right)^{2}}(\partial \varphi)^{2}-V(\varphi) .
$$

We introduce a canonically normalized field $\chi$ satisfying

$$
\left(\frac{d \chi}{d \varphi}\right)^{2}=\frac{2 u-\frac{\varphi^{2}}{6} \xi(1-\xi)}{\left(2 u-\frac{1}{6}(1-\xi) \varphi^{2}\right)^{2}}
$$

Integrating (while choosing $u=1 / 2$ ), we obtain the canonically normalized field $\chi$ as a function of $\varphi$,

$$
\begin{aligned}
\chi= & \sqrt{6} \tanh ^{-1}\left(\frac{(1-\xi) \varphi}{\sqrt{6\left(1-\frac{\xi(1-\xi) \varphi^{2}}{6}\right)}}\right) \\
& -\sqrt{\frac{6 \xi}{1-\xi}} \sinh ^{-1}\left(\sqrt{\xi\left(\frac{1-\xi}{6}\right) \varphi}\right) .
\end{aligned}
$$

\section{A. The $\mu$ problem and a nonzero VEV for the $S$ field}

In Sec. II we discussed the basic features of the model under consideration and introduced an $R$ symmetry to constrain the superpotential. In the present section we discuss the appearance of new contributions, including those coming from supersymmetry-breaking effects.

The supersymmetric Higgs mass parameter $\mu$ (associated with the so-called $\mu$ problem [37]) can be generated when the $S$ field acquires a nonzero VEV not much higher than the electroweak scale. Contributions from the soft SUSYbreaking terms, although negligible during inflation, may generate the required nonzero $\mathrm{VEV},\langle S\rangle \neq 0$ [34].

Furthermore, in principle, we can include a constant term $W_{0}$ in the superpotential (2.2) which gives rise to a gravitino mass [38-40] through the formula $m_{3 / 2}=e^{K / 2}\left\langle W_{0}\right\rangle$. The magnitude of the constant $W_{0}$ is model dependent and its presence in the superpotential has important implications on the dynamics of the effective theory. In the present model, however, $W_{0}$ violates the $R$ symmetry of the superpotential (2.2) which has been imposed in order to ensure linearity on $S$ and thereby the gravitino cannot acquire a mass within this context. Nevertheless, an alternative way to generate a nonzero $m_{3 / 2}$ that is rather appropriate here is the mechanism described in Ref. [34] (see also Ref. [41]). This relies on the soft SUSY-breaking superpotential terms mentioned above which shift the VEV of the field $S$ to a nonzero value $\langle S\rangle \neq 0$. This is sufficient to solve the $\mu$ problem and - at the same time-generate a nonzero mass for the gravitino.

Next, we focus on the scalar potential. Inserting the values $H^{0}= \pm M, N^{0}=0$, and $H_{u}^{0}=H_{d}^{0}=0$, the total potential takes the form

$$
\begin{aligned}
V_{t}(S)= & \frac{2 \kappa^{2} M^{2} S^{2}}{\left(2 u-\frac{2 M^{2}(1-\xi)+S^{2}}{3}\right)}+m_{S}^{2} S^{2}+\kappa A_{\kappa} M^{2} S \\
& -a_{S} \kappa M^{2}+\text { H.c. }
\end{aligned}
$$

Assuming $S \ll 1$, we may expand the first term of Eq. (3.18) in powers of $S$. Neglecting terms higher than $S^{2}$ in the expansion, the potential can be expressed in terms of the gravitino mass $m_{3 / 2}$ as follows:

$V_{t}(S)=\frac{2 \kappa^{2} M^{2} S^{2}}{\left(2 u-\frac{2 M^{2}(1-\xi)}{3}\right)^{2}}+m_{S}^{2} S^{2}+2 b \kappa m_{3 / 2} M^{2} S$,

where we used $\left|a_{s}\right|+\left|A_{\kappa}\right|=2 b m_{3 / 2}$, while the $m_{S}^{2}$ term has been neglected since $m_{S}^{2} \ll M^{2}$. Minimization of the potential now gives

$$
\sigma \equiv\langle S\rangle=-\frac{b m_{3 / 2}}{2 \kappa}\left(2 u-\frac{2 M^{2}(1-\xi)}{3}\right)^{2} .
$$

The second derivative with respect to $S$,

$$
\left.\frac{d^{2} V_{t}}{d S^{2}}\right|_{\sigma}=\frac{4 \kappa^{2} M^{2}}{\left(2 u-\frac{2 M^{2}(1-\xi)}{3}\right)^{2}}>0,
$$

is always positive, and the potential acquires a minimum for $\langle S\rangle \neq 0$. Hence, the $\mu$ parameter now is dynamically generated as long as $S$ receives a nonzero VEV,

$$
\mu=\lambda \sigma=-\frac{\lambda b m_{3 / 2}}{2 \kappa}\left(2 u-\frac{2 M^{2}(1-\xi)}{3 u}\right)^{2} .
$$

Finally, for $\langle S\rangle \neq 0$ the effective potential takes the following form:

$$
\begin{aligned}
V(\varphi)= & \frac{\kappa^{2}\left(M^{2}-\frac{\varphi^{2}}{4}\right)^{2}+\frac{\kappa^{2} \sigma^{2} \varphi^{2}}{2}}{\left(2 u-\left(\frac{1-\xi}{6}\right) \varphi^{2}-\frac{\sigma^{2}}{3}\right)^{2}}+\frac{m^{2}}{2} \varphi^{2} \\
& -\frac{b^{2} M^{2} m_{3 / 2}^{2}}{2}\left(2 u-\frac{2 M^{2}(1-\xi)}{3}\right)^{2} .
\end{aligned}
$$

So, the Lagrangian in this case reads 
$\mathcal{L}(\varphi)=\frac{2 u-\frac{\varphi^{2}}{6} \xi(1-\xi)}{2\left(2 u-\frac{1}{6}(1-\xi) \varphi^{2}-\frac{\sigma^{2}}{3}\right)^{2}}(\partial \varphi)^{2}-V(\varphi)$,

and the canonically normalized field $\chi$ now satisfies the equation

$$
\chi^{\prime} \equiv\left(\frac{d \chi}{d \varphi}\right)=\sqrt{\frac{2 u-\frac{\varphi^{2}}{6} \xi(1-\xi)}{\left(2 u-\frac{1}{6}(1-\xi) \varphi^{2}-\frac{\sigma^{2}}{3}\right)^{2}}} .
$$

We turn now to a numerical analysis and compute the various observables related to inflation.

\section{ANALYSIS}

In this section we analyze the implications of the model and discuss its predictions regarding the various cosmological observables. We pay particular attention to leptogenesis, large- $r$ solutions, and gravitational waves. Before presenting numerical predictions, we briefly review the basic results of the slow-roll assumption.

\section{A. Slow-roll approximation}

The inflationary slow-roll parameters are given by

$$
\begin{aligned}
& \epsilon=\frac{1}{2}\left(\frac{V^{\prime}(\varphi)}{V(\varphi) \chi^{\prime}(\varphi)}\right)^{2}, \\
& \eta=\left(\frac{V^{\prime \prime}(\varphi)}{V(\varphi)\left(\chi^{\prime}(\varphi)\right)^{2}}-\frac{V^{\prime}(\varphi) \chi^{\prime \prime}(\varphi)}{V(\varphi)\left(\chi^{\prime}(\varphi)\right)^{3}}\right) .
\end{aligned}
$$

The third slow-roll parameter is

$$
\begin{aligned}
s^{2}= & \left(\frac{V^{\prime}(\varphi)}{V(\varphi) \chi^{\prime}(\varphi)}\right)\left(\frac{V^{\prime \prime \prime}(\varphi)}{V(\varphi)\left(\chi^{\prime}(\varphi)\right)^{3}}-3 \frac{V^{\prime \prime}(\varphi) \chi^{\prime \prime}(\varphi)}{V(\varphi)\left(\chi^{\prime}(\varphi)\right)^{4}}\right. \\
& \left.+3 \frac{V^{\prime}(\varphi)\left(\chi^{\prime \prime}(\varphi)\right)^{2}}{V(\varphi)\left(\chi^{\prime}(\varphi)\right)^{5}}-\frac{V^{\prime}(\varphi) \chi^{\prime \prime \prime}(\varphi)}{V(\varphi)\left(\chi^{\prime}(\varphi)\right)^{4}}\right),
\end{aligned}
$$

where a prime denotes a derivative with respect to $\varphi$. The slow-roll conditions are $\epsilon \ll 1,|\eta| \ll 1$, and $s^{2} \ll 1$, while the tensor-to-scalar ratio $r$, the scalar spectral index $n_{s}$, and the running of the spectral index $\frac{d n_{s}}{d \ln k}$ are given by

$$
\begin{aligned}
r & \simeq 16 \epsilon, \quad n_{s} \simeq 1+2 \eta-6 \epsilon, \\
\frac{d n_{s}}{d \ln k} & \simeq 16 \epsilon \eta-24 \epsilon^{2}+2 s^{2} .
\end{aligned}
$$

The number of $e$-folds is given by

$$
\begin{aligned}
N_{l} & =\int_{\varphi_{e}}^{\varphi_{l}}\left(\frac{V(\varphi) \chi^{\prime}(\varphi)}{V^{\prime}(\varphi)}\right) d \varphi \\
& =54+\frac{1}{3} \ln \left[\frac{T_{r}}{10^{9} \mathrm{GeV}}\right]+\frac{1}{3} \ln \left[\frac{V\left(\varphi_{l}\right)^{1 / 4}}{10^{16} \mathrm{GeV}}\right] .
\end{aligned}
$$

In the above equation, $l$ is the comoving scale after crossing the horizon and $\varphi_{l}$ is the field value at $l$. Also, $\varphi_{e}$ is the field at the end of inflation (i.e., when $\epsilon=1$ ) and $T_{r}$ is the reheating temperature, which will be discussed in the following section. Furthermore, in the analysis the constraints from the amplitude of the curvature perturbation $\Delta_{R}$ should be implemented:

$$
\Delta_{R}^{2}=\frac{V(\varphi)}{24 \pi^{2} \epsilon(\varphi)}
$$

\section{B. Reheating temperature and nonthermal leptogenesis}

A successful inflationary scenario should be followed by thermalization (reheating) triggered by the inflaton decay through its couplings to SM fields and, in particular as far as the present model is concerned, the right-handed neutrinos. This coupling, however, is subjected to constraints, associated with the issue of gravitino overproduction during thermalization $[42,43]$. The abundance of the latter depends on the decay width of the inflaton which is related to the reheating temperature. On the other hand, there is an upper bound on the abundance of dark matter originating from the decay of gravitinos which is converted to the upper bound of the reheating temperature $T_{r} \lesssim 10^{6}-10^{11} \mathrm{GeV}$. In particular, a more precise constraint on $T_{r}$ depends on the SUSY-breaking mechanism and the gravitino mass $m_{3 / 2}$. For gravity-mediated SUSYbreaking models with unstable gravitinos of mass $m_{3 / 2} \simeq(0.1-1) \mathrm{TeV}$, the reheating temperature bound is $T_{r} \lesssim 10^{6}-10^{9} \mathrm{GeV}[44,45]$, while in the case of stable gravitinos it increases to $T_{r} \lesssim 10^{10} \mathrm{GeV}$ [46]. In gaugemediated models the reheating temperature is generally more severely constrained, although $T_{r} \sim 10^{9}-10^{10} \mathrm{GeV}$ is possible for $m_{3 / 2} \simeq 5-100 \mathrm{GeV}$ [47]. Finally, the anomaly-mediated symmetry-breaking scenario may allow gravitino masses much heavier than a few $\mathrm{TeV}$, thus accommodating a reheat temperature as high as $10^{11} \mathrm{GeV}$ [48]. In the present work we focus on a gravity-mediated SUSY-breaking scenario, and in the next section we briefly discuss different cases for the lightest supersymmetric particle (LSP).

The transition to the radiation epoch is controlled by the inflaton mass and its decay channels. After the end of inflation, the inflaton starts oscillating around the minimum. For the following analysis we define the canonically normalized inflaton

$$
\delta \chi=\left\langle\chi^{\prime}\right\rangle \delta \varphi \quad \text { with } \quad \delta \varphi=(\varphi-2 M),
$$

where $\chi^{\prime}$ is defined in Eq. (3.16). The noncanonical normalized fields $\varphi$ and $H$ are related to each other by $H=\varphi / 2$. The magnitude of $\left\langle\chi^{\prime}\right\rangle$ at the minimum $\varphi^{0}=2 M$ reads 


$$
\left.\left\langle\chi^{\prime}\right\rangle\right|_{\varphi^{0} \rightarrow 2 M}=\frac{2 u-\frac{2 M^{2}}{3} \xi(1-\xi)}{\left(2 u-\frac{2 M^{2}}{3}(1-\xi)-\frac{\sigma^{2}}{3}\right)^{2}},
$$

and the inflaton acquires a mass given by

$$
m_{\mathrm{inf}}^{2}=\frac{d^{2} V(\varphi)}{d \chi^{2}}=\left(\frac{V^{\prime \prime}(\varphi)}{\left(\chi^{\prime}\right)^{2}}-\frac{V^{\prime}(\varphi) \chi^{\prime \prime}}{\left(\chi^{\prime}\right)^{3}}\right)_{\varphi^{0} \rightarrow 2 M} .
$$

Due to the superpotential terms $\beta \bar{H} N^{c} N^{c}$ and $\lambda S H_{u} H_{d}$, the possible decay channels of the fields $\delta \chi, S$ are to a pair of right-handed neutrinos and sneutrinos $\left(N^{c}, \tilde{N}_{c}\right)$ and to $H_{u}$ and $H_{d}$, respectively. The relevant Lagrangian terms are

$$
\mathcal{L}_{\delta \chi \rightarrow N^{c} N^{c}}=-\frac{1}{2} e^{K / 2} W_{, N^{c} N^{c}} N^{c} N^{c} \rightarrow-\alpha_{N^{c}} \delta \chi N^{c} N^{c},
$$

with $W$ and $K$ as defined in Eqs. (3.1) and (3.2), while $W_{, N^{c}, N^{c}}$ is the second derivative of $W$ with respect to the field $N^{c}$. Finally, $\alpha_{N^{c}}$ is the effective coupling of inflaton decay to right-handed neutrino fields, which is defined as

$$
\alpha_{N^{c}}=\frac{\beta}{4 \sqrt{\left(2 u-\frac{2}{3} M^{2}(1-\xi)-\frac{\sigma^{2}}{3}\right)\left(2 u-\frac{2}{3} M^{2} \xi(1-\xi)\right)}} .
$$

Hence, the decay width is [41]

$$
\Gamma_{\delta \chi \rightarrow N^{c} N^{c}}=\frac{1}{16 \pi} \alpha_{N^{c}}^{2} m_{\mathrm{inf}}\left(1-\frac{4 M_{N^{c}}^{2}}{m_{\mathrm{inf}}^{2}}\right)^{3 / 2},
$$

where $M_{N}$ is the Majorana mass

$$
\frac{\beta * M}{\left(2 u-\frac{2}{3} M^{2}(1-\xi)-\frac{\sigma^{2}}{3}\right)^{3 / 2}} .
$$

Similarly, for the inflaton decay to $H_{u}$ and $H_{d}$, the relevant Lagrangian is

$$
\mathcal{L}_{\delta \chi \rightarrow H_{u} H_{d}}=-e^{K} K_{, S S^{*}}\left|W_{, S}\right|^{2}=-\alpha_{h} \delta \chi H_{u} H_{d}
$$

and the effective coupling for inflaton decay to Higgs fields is

$$
\alpha_{h}=\frac{\kappa \lambda M\left(2 u-\frac{2 M^{2}(1-\xi)}{3}\right)}{\sqrt{\left(2 u-\frac{2}{3} M^{2} \xi(1-\xi)\right)}\left(2 u-\frac{2 M^{2}(1-\xi)+\sigma^{2}}{3}\right)^{4}} .
$$

Thus, the decay width in this case is [41]

$$
\Gamma_{\delta \chi \rightarrow H_{u} H_{d}}=\frac{\alpha_{h}^{2}}{8 \pi} m_{\mathrm{inf}} .
$$

The reheating temperature $T_{r}$ for the $U(1)_{B-L}$ extended MSSM spectrum is given by [41]

$$
T_{r}=\left(\frac{72}{5 \pi^{2} g^{*}}\right)^{1 / 4} \sqrt{\Gamma} \quad \text { with } \quad \Gamma=\Gamma_{\delta \chi \rightarrow N^{c} N^{c}}+\Gamma_{\delta \chi \rightarrow H_{u} H_{d}}
$$

The two conditions

$$
\frac{m_{\text {inf }}}{2} \geq M_{N}, \quad M_{N} \geq 10 T_{r}
$$

ensure a successful reheating process with nonthermal leptogenesis. These two conditions put strong bounds on the reheating temperature.

Next, we present numerical predictions of the model with respect to the slow-roll parameters and the reheating constraints.

\section{Large-r solutions and observable gravity waves}

Primordial gravity waves are associated with the tensorto-scalar ratio $r$ which will be measured with higher accuracy in the next-generation experiments such as PRISM, LiteBIRD, PIXIE, and CORE [49-52]. Future measurements are expected to reach values as low as $r \sim 5 \times 10^{-4}$.

Here, we have performed numerical calculations to provide predictions for the ratio $r$ as well as other observables. Figure 2 shows ranges for the various parameters involved in the effective potential. The plot in the top panel shows $r$ as a function of the right-handed neutrino coupling $\beta$, for various values of the reheating temperature $T_{r}$. The various curves start with $m=1 \mathrm{TeV}$ from the left and stop when $r$ has the value $r=0.11$. The reheating temperature varies from $T_{r}=10^{9} \mathrm{GeV}$ (dark green curve at the top) to $T_{r} \simeq 10^{5.8} \mathrm{GeV}$ (blue curve) as $\beta$ decreases. The spectral index is fixed at $n_{s}=0.9655$ (central value) and the GUT-scale mass parameter is fixed at $M=2 \times 10^{16} \mathrm{GeV}$. The remaining plots show the predictions for the parameters $m, \lambda, \kappa$, and $\xi$. Furthermore, we consider $b=1$ and choose $|\mu|=m_{3 / 2}$ for each curve. As can be observed in Fig. 2, the reheating bound (4.14) is satisfied.

Figure 3 (left panel) shows the variations of the inflaton mass with respect to $r$. The plot shows that the mass of the inflaton lies in the range $9.9 \times 10^{11} \leq m_{\mathrm{inf}} \leq 9.1 \times 10^{12} \mathrm{GeV}$. The plot on the right shows the predictions for the righthanded heavy Majorana scale $M_{N}$ as a function of $r$. Depending on the value of the reheating temperature, $M_{N}$ varies from $10^{8}$ up to $10^{12} \mathrm{GeV}$, which is heavy enough to realize small neutrino masses via the seesaw mechanism. In all of the cases, the results are in accordance with the bounds given in Eq. (4.14).

Finally, Fig. 4 (left panel) shows the width of the inflaton decay to Majorana neutrinos with respect to $r$. In the right panel we plot the decay rate of the inflaton field to Majorana neutrinos vs the decay rate to the Higgs fields. 

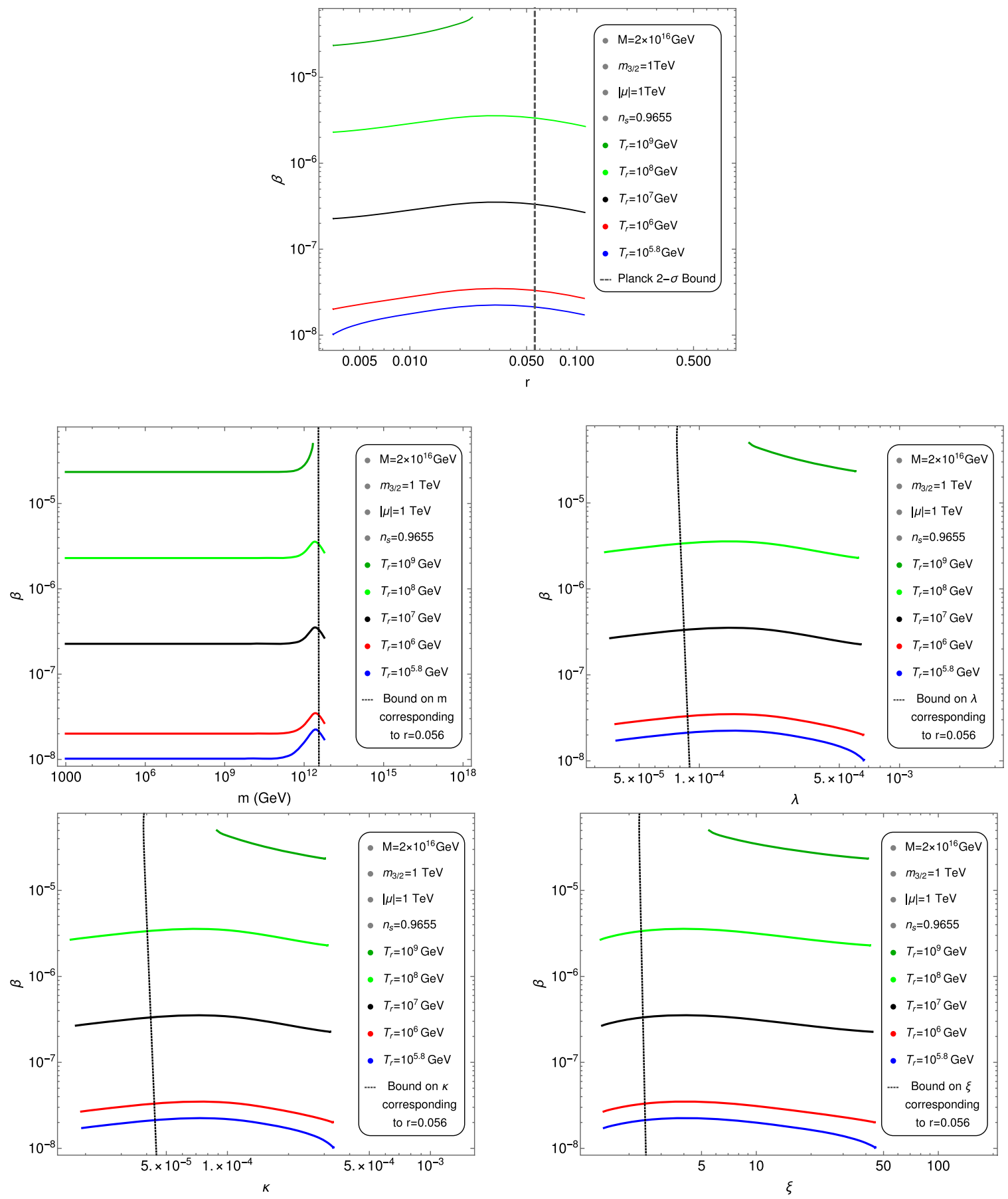

FIG. 2. The top panel shows predictions for the tensor-to-scalar ratio $r$ as a function of the coupling constant $\beta$ for various values of the reheating temperature $T_{r}$. Each curve corresponds to a fixed value of $T_{r}$. For all of the curves we fix the GUT scale at $M=2 \times 10^{16} \mathrm{GeV}$, the spectral index $n_{s}=0.9655$ (central value), and the gravitino mass and the Higgsino mass parameter at $m_{3 / 2}=\mu=1 \mathrm{TeV}$. The lower panels show the ranges for the other parameters $(m, \lambda, \kappa, \xi)$ involved in the analysis with respect to the $(\beta, r)$ plot.

The dominance of the inflaton decay to Majorana neutrinos compared to Higgs fields is reflected in this figure.

\section{Varying the SUSY scale: Implications}

The results obtained show that the range of the tensor-toscalar ratio is $r \approx\left[0.11-10^{-3}\right]$. The Planck measurement of $r$ is up to $r=0.056$ at $95 \%$ C.L. which is shown by the gray dashed line in Fig. 2. In the lower-left panel of Fig. 2 the parameter $\beta$ vs $m$ is plotted. The gray line puts the cutoff for the soft breaking mass at $m_{\text {SUSY }} \leq 5 \times 10^{12} \mathrm{GeV}$. For $m_{\text {SUSY }}<10^{6} \mathrm{TeV}$, the tensor-to-scalar ratio is around $r \simeq 10^{-3}$. For high-scale (split) SUSY where 

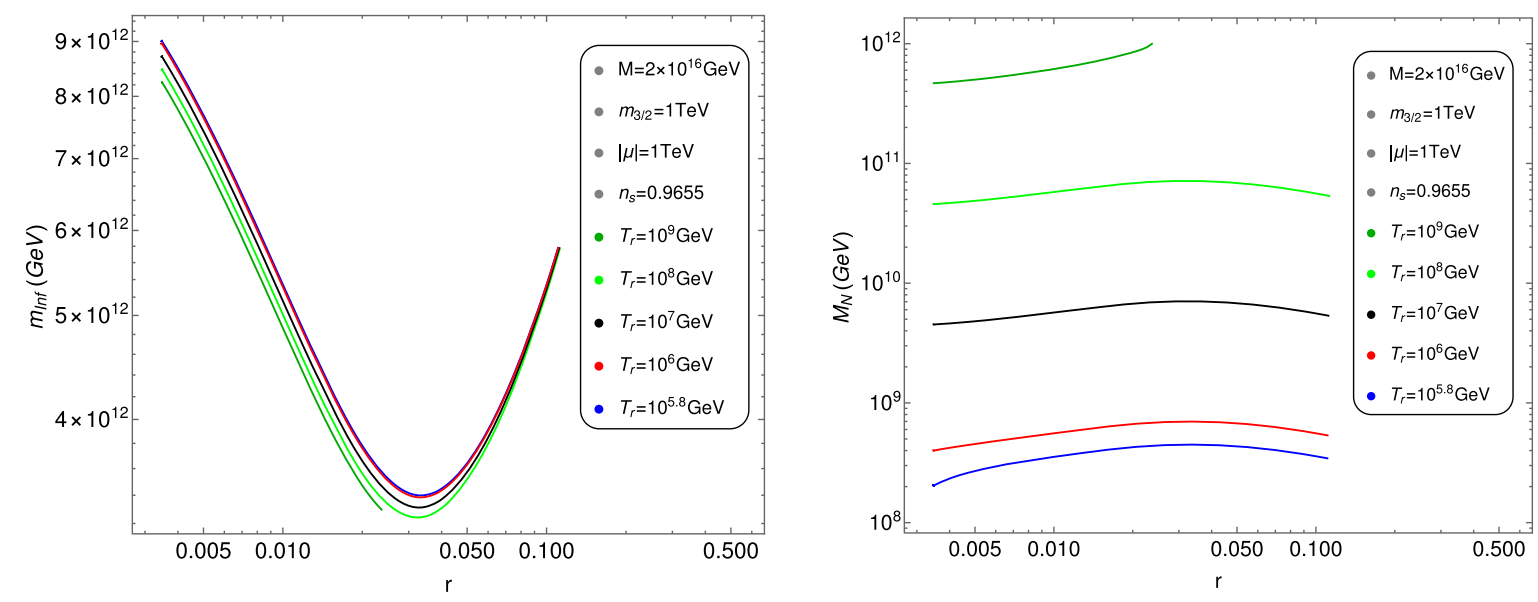

FIG. 3. The plot on the left shows variations of the inflaton mass $m_{\text {inf }}$ as a function of the tensor-to-scalar ratio $r$. The right panel shows $M_{N}$ vs $r$. Each curve corresponds to a different value for the reheating temperature $T_{r}$.
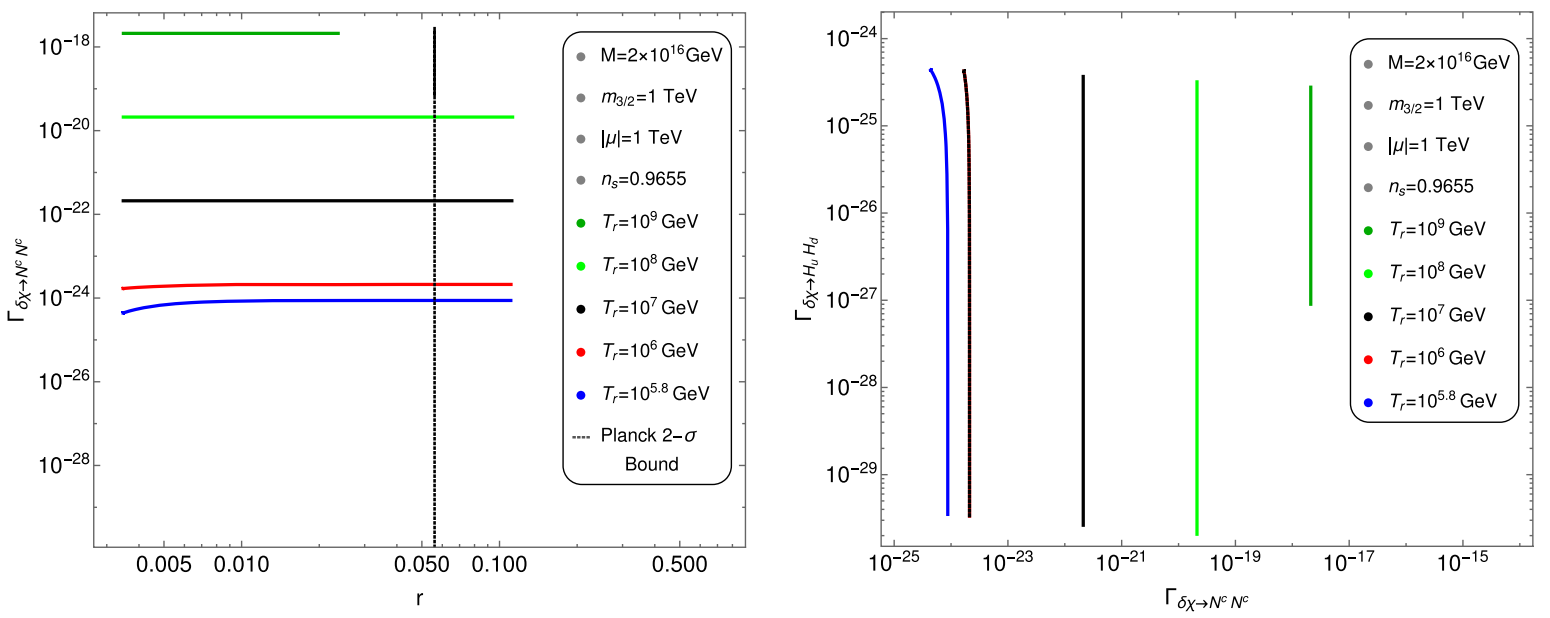

FIG. 4. Left: tensor-to-scalar ratio $r$ vs the decay width of the inflaton to right-handed neutrino fields. Right: decay width of the inflaton to right-handed neutrinos vs the decay width of the inflaton to Higgs fields.

$m_{\text {SUSY }} \geq 10^{6} \mathrm{TeV}$, we obtain a tensor-to-scalar ratio $r \simeq$ $10^{-2}$ which is in very good agreement with the measurements of $r$ from Planck and in next-generation experiments.

For TeV-scale SUSY, big bang nucleosynthesis (BBN) imposes new bounds. In our model, a TeV-scale SUSY scenario is very consistent with our results and the observed BBN bounds. Similarly, for split SUSY and high-scale SUSY scenarios the reheating temperature is bounded by $\Omega_{\mathrm{LSP}}$, which we discuss in detail in the following sections.

\section{GRAVITINO DARK MATTER}

In this section we briefly discuss whether there are regions of the parameter space consistent with a gravitino dark matter (DM) component. According to Refs. [53,54], one may consider the cases of (i) a stable LSP gravitino, (ii) a unstable long-lived gravitino with mass $m_{3 / 2}<$ $25 \mathrm{TeV}$, and (iii) an unstable short-lived gravitino with mass $m_{3 / 2}>25 \mathrm{TeV}$. In the first case, the gravitino is a potential DM candidate and, assuming it is thermally produced, its relic density is estimated to be [55]

$\Omega_{3 / 2}^{2}=0.08\left(\frac{T_{r}}{10^{10} \mathrm{GeV}}\right)\left(\frac{m_{3 / 2}}{1000 \mathrm{GeV}}\right)\left(1+\frac{m_{\tilde{g}^{2}}}{3 m_{3 / 2}^{2}}\right)$,

where $m_{\tilde{g}}$ is the gluino mass parameter and for simplicity $m_{3 / 2}=m=|\mu|$ is assumed. ${ }^{1}$ A stable LSP gravitino

${ }^{1}$ Equation (5.1) contains only the dominant QCD contributions for the gravitino production rate. In principle, there are extra contributions coming from the electroweak sector, as mentioned in Ref. [56,57] and recently revised in Ref. [58]. If we consider these types of contributions in our analysis, we estimate that (depending on the gaugino universality condition) our results will differ by $\sim(10-15) \%$. 


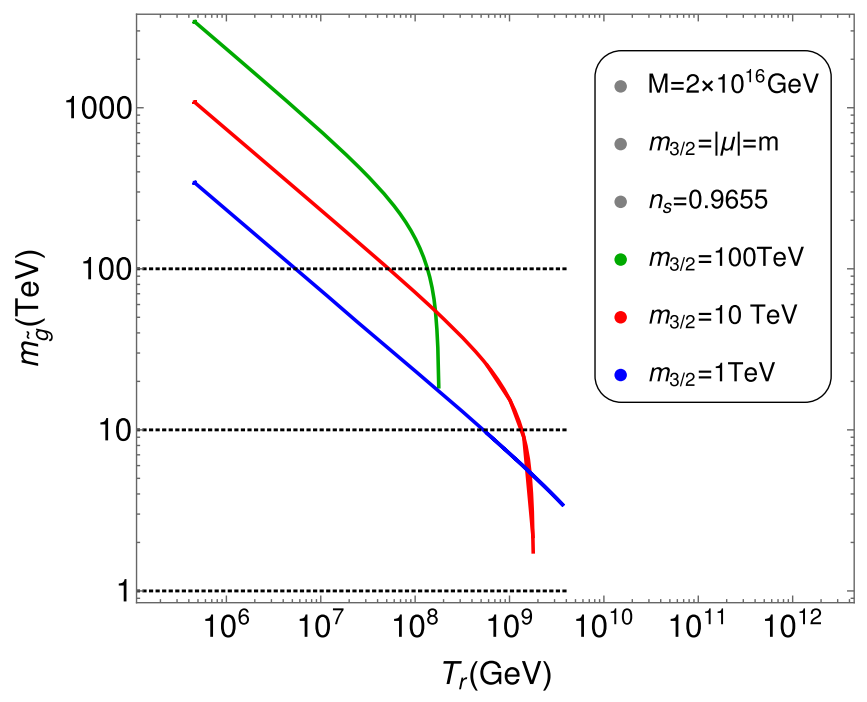

FIG. 5. The case of an LSP gravitino with mass $m_{3 / 2}=1$, $10,100 \mathrm{TeV}$. The corresponding gluino masses $m_{\tilde{g}}$ are shown by the solid blue, red, and green lines with $n_{s}=0.9655$ and $M=2 \times 10^{16} \mathrm{GeV}$.

requires $m_{\tilde{g}}>m$, while current LHC bounds on the gluino mass are around $2.2 \mathrm{TeV}[59,60]$. Taking the lower bound of the relic abundance $\Omega_{h}^{2}=0.144$ [5], Fig. 5 shows the range of $m_{\tilde{g}}$ as a function of the reheating temperature $T_{r}$ for representative values of $m_{3 / 2}$.

It is seen from Fig. 5 that for $m_{3 / 2}=1 \mathrm{TeV}$ (blue curve) the gravitino is the LSP since the kinematic condition $m_{3 / 2}<m_{\tilde{g}}$ is always true. For $m_{3 / 2}=10 \mathrm{TeV}$ (red curve) the gravitino is the LSP in the regions with $m_{\tilde{g}}>10 \mathrm{TeV}$. Below $m_{\tilde{g}}=10 \mathrm{TeV}$ (dotted black line) the gravitino is the next-to-lightest supersymmetric particle (NLSP). A similar description holds for the case with $m_{3 / 2}=100 \mathrm{TeV}$ (green curve). Therefore, we see that there are regions in the parameter space where the gravitino is the LSP, and as such it contributes to DM.

Next, we consider the possibility where the gravitino is not the LSP. The decay of the gravitino occurs after the freeze-out epoch of the lightest neutralino, which will play the role of the LSP. The lightest neutralino has two origins: one is thermal and the other is nonthermal. Thermal production consists of the standard freeze-out mechanism of weakly interacting massive particles, whereas nonthermal production deals with the decay product of the gravitino produced during the reheating process [61]. However, since the density of the thermal relic is strongly model dependent, ${ }^{2}$ we do not take its effect into account in the calculation of the density parameter. Here we distinguish two cases of gravitino decay: either a longlived or a short-lived. For a long-lived gravitino with mass

\footnotetext{
${ }^{2}$ For a detailed analysis with emphasis on the DM phenomenology of the model, see Ref. [33].
}

$m_{3 / 2}<25 \mathrm{TeV}$, its lifetime is about $\tilde{\tau} \gtrsim 1 \mathrm{~s}$. However, in this case we encounter the cosmological gravitino problem [42] that originates due to the fast decay of the gravitino, which may affect the light nuclei abundances and thereby ruin the success of BBN theory. To avoid this problem, one has to take into account the BBN bounds on the reheating temperature, which are [61]

$T_{r} \lesssim 3 \times\left(10^{5}-10^{6}\right) \mathrm{GeV} \quad$ for $m_{3 / 2}=1 \mathrm{TeV}$,

$T_{r} \lesssim 2.5 \times 10^{9} \mathrm{GeV}$ for $m_{3 / 2}=10 \mathrm{TeV}$.

We see from Fig. 5 that a long-lived gravitino with $m_{3 / 2}=1 \mathrm{TeV}$ is not a viable scenario because it becomes the NLSP for a reheating temperature $T_{r} \geq 2 \times 10^{9} \mathrm{GeV}$. Nevertheless, for $m_{3 / 2} \geq 10 \mathrm{TeV}$ a long-lived gravitino scenario is viable and consistent with the BBN bounds (5.2) for the reheating temperature.

In order to discuss the scenario of a short-lived gravitino (for instance, with mass $m_{3 / 2}=100 \mathrm{TeV}$ ), we recall that the gravitino decays before $\mathrm{BBN}$, and as a result the BBN bounds on the reheating temperature are not effective. In this case, the gravitino decays into the LSP neutralino $\tilde{\chi}_{1}^{0}$. The neutralino abundance is given by

$$
\Omega_{\tilde{\chi}_{1}^{0}} h^{2} \simeq 2.8 \times 10^{11} \times Y_{3 / 2}\left(\frac{m_{\tilde{\chi}_{1}^{0}}}{1 \mathrm{TeV}}\right)
$$

where $Y_{3 / 2}$ is the gravitino yield and is defined as

$$
Y_{3 / 2} \simeq 2.3 \times 10^{-12}\left(\frac{T_{r}}{10^{10} \mathrm{GeV}}\right)
$$

As we know, the LSP neutralino density produced by gravitino decay should not exceed the observed DM relic density. Choosing the upper bound of the relic abundance $\Omega_{\tilde{\chi}_{1}^{0}} h^{2}=0.126$ and using Eqs. (5.4) and (5.3), we find a relation between the reheating temperature $T_{r}$ and $m_{\tilde{\chi}_{1}^{0}}$, which is

$$
m_{\tilde{\chi}_{1}^{0}} \simeq 19.6\left(\frac{10^{11} \mathrm{GeV}}{T_{r}}\right)
$$

For the gravity-mediation scenario, $m_{\tilde{\chi}_{1}^{0}} \geq 18 \mathrm{GeV}$ [62], which can easily satisfied. For a gravitino with $m_{3 / 2}=$ $100 \mathrm{TeV}$, the bounds on the LSP neutralino mass derived as in Eq. (5.5) are

$$
5.21 \times 10^{2} \leq m_{\tilde{\chi}_{1}^{0}}(\mathrm{GeV}) \leq 4.25 \times 10^{6} .
$$

Figure 6 shows the plot of $m_{\tilde{\chi}_{1}^{0}}$ vs $T_{r}$. Therefore, the shortlived gravitino scenario is also a viable possibility in this model. 


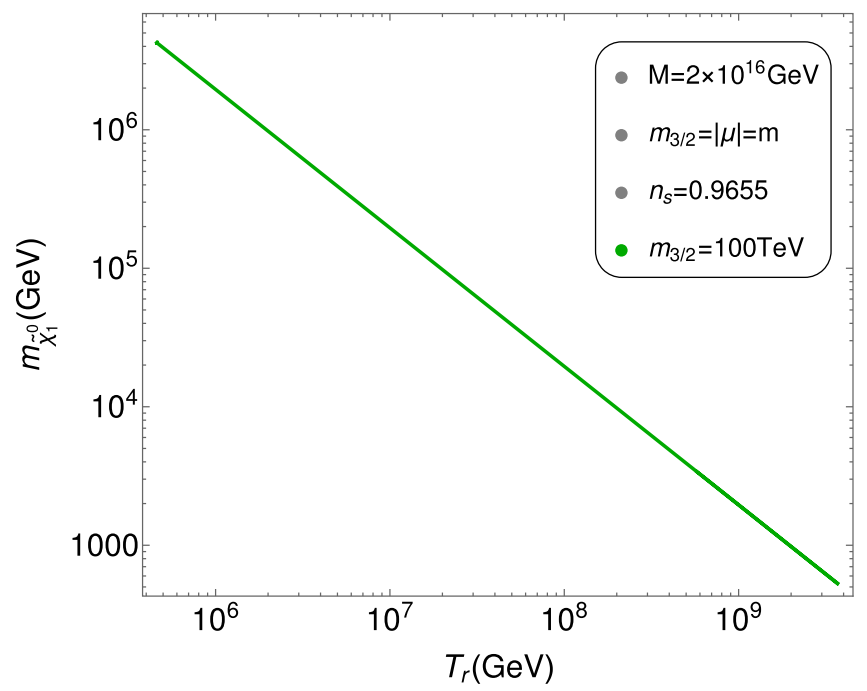

FIG. 6. Short-lived gravitino with mass $m_{3 / 2}=100 \mathrm{TeV}$ and the corresponding neutralino masses $m_{\tilde{\chi}_{1}^{0}}$ with $n_{s}=0.9655$ and $M=2 \times 10^{16} \mathrm{GeV}$.

\section{QUANTUM TUNNELING FROM FALSE TO TRUE VACUUM}

A careful consideration of the previous analysis reveals regions of the parameter space where, in addition to the global minimum $V_{\min }\left(\varphi_{0}\right)$ (true vacuum), a second (higher) ground state evolves at some point $\varphi \neq \varphi_{0}$, usually called the false vacuum. Depending on the initial conditions, the inflaton may roll down the slope of the potential towards the false vacuum and subsequently make the transition to the true minimum. Such transitions can be described by Euclidean instantons in a way suggested by Coleman and De Luccia (CdL) [63].

In order to discuss the dynamics of the vacuum in this new context, we rewrite the noncanonical normalized potential of Eq. (3.14) in the form

$$
V(\Phi)=\frac{1}{2} \mu^{2} \Phi^{2}+\frac{\lambda}{4} \frac{\left(1-\Phi^{2}\right)^{2}}{\left(1-\gamma \Phi^{2}\right)^{2}}
$$

where we have made the following redefinitions:

$$
\begin{aligned}
\Phi & =\frac{\varphi}{2 M}, & \gamma=\frac{(1-\xi) M^{2}}{3 u}, \\
\mu & =2 m M, & \lambda=\left(\frac{\kappa M^{2}}{u}\right)^{2} .
\end{aligned}
$$

Since $m<M<1$, the parameters $\mu$ and $\lambda$ are expected to be less than unity.

For the special case $\xi=1$ we have $\gamma=0$, and the potential simplifies to

$$
V(\Phi)=\frac{1}{2} \mu^{2} \Phi^{2}+\frac{\lambda}{4}\left(1-\Phi^{2}\right)^{2} .
$$

Similarly, in the large value of $|\xi|$ we have $\gamma=|\xi| M^{2} /(3 u)$. The form of $V$ in Eq. (6.3) represents a double-well potential, with the minima at a radius $\Phi_{0}=\sqrt{1-\frac{\mu^{2}}{\lambda}}$ and local maximum at $\Phi=0$. In this new parametrization the general case corresponds to $\gamma \neq 0$ or $\xi \neq 1$. The extrema are determined by

$\frac{d V(\Phi)}{d \Phi}=0 \Rightarrow \mu^{2} \Phi\left(1-\frac{\lambda}{\mu^{2}} \frac{(1-\gamma)\left(1-\Phi^{2}\right)}{\left(1-\gamma \Phi^{2}\right)^{3}}\right)=0$.

There is an obvious extremum (in fact, a minimum) at $\Phi=0$. The parentheses include a cubic equation with respect to $\Phi^{2}$, implying three roots for $\Phi^{2}$. There are either three real roots or a single one depending on the values of the parameter $\gamma$ and the ratio $\mu^{2} / \lambda=R$. The case of the metastable de Sitter vacuum corresponds to the case of three real roots, since there should be a minimum and a maximum before $\Phi=0$ and $\Phi=\infty$. To simplify the cubic equation, we use the redefinitions

$y=1-\gamma \Phi^{2} \rightarrow \Phi^{2}=\frac{1-y}{\gamma}, \quad$ for $\gamma \neq 0, \quad$ or $\xi \neq 1$.

Then Eq. (6.4) is written as

$$
y^{3}+p y+q=0,
$$

with

$p=-\frac{1-\gamma}{\gamma R}, \quad q=-(1-\gamma) p=\frac{(1-\gamma)^{2}}{\gamma R}=\frac{a^{2}}{\gamma R}$.

Three real roots exist if the discriminant $4 p^{3}+27 q^{2}<0$, implying

$$
(a / \gamma R)^{3}(-1+27 a R \gamma / 4)<0 .
$$

Substituting $y=v \cos \theta$ and comparing with the trigonometric identity $\cos (3 \theta)=4 \cos ^{3}(\theta)-3 \cos (\theta)$, we find

$$
y=2 \sqrt{\frac{-p}{3}} \cos \left(\frac{1}{3} \arccos \left(\frac{3 q}{p} \sqrt{-\frac{3}{4 p}}\right)-\frac{2 n \pi}{3}\right), n=0,1,2
$$

or

$$
y=2 \sqrt{\frac{1-\gamma}{3 \gamma R}} \cos \left(\frac{1}{3} \arccos \left(-\frac{3}{2} \sqrt{3 R \gamma(1-\gamma)}\right)-\frac{2 n \pi}{3}\right),
$$

$n=0,1,2$.

Real roots exist when the argument of arccos is less than one, 


$$
\left|-\frac{3}{2} \sqrt{3 R \gamma(1-\gamma)}\right| \leq 1
$$

which is just the constraint on the discriminant. Assuming $0<R<1$ and using Eq. (6.8), we can put bounds on the various parameters of the potential. The above inequality can be simplified as

$$
\gamma^{2}-\gamma+\frac{4}{27 R} \geq 0
$$

For real roots of $\gamma$ the discriminant must be greater than or equal to zero, which means $1-\frac{16}{27 R} \geq 0$ or $R \geq \frac{16}{27}$. This constrains $R$ in the range $16 / 27 \leq R<1$, or in terms of $\mu$ and $\lambda$ we have that

$$
\mu^{2}<\lambda \leq \frac{27}{16} \mu^{2}
$$

The bound on $R$ also helps us to obtain a lower bound on $\gamma$, which is $\gamma \geq \frac{1}{2}\left(1-\sqrt{\frac{11}{27}}\right)$. The upper value of $\gamma$ basically separates the potential into two regions. Then, Eq. (3.16) between the canonically and noncanonically normalized fields with respect to the new parametrization can be written as

$$
\frac{d X}{d \Phi}=\sqrt{\frac{1-\gamma \Phi^{2}\left(1-\frac{3 u}{M^{2}} \gamma\right)}{2 u\left(1-\gamma \Phi^{2}\right)^{2}}}
$$

where $X=\chi / 2 M$ is the normalized field.

We solve Eq. (6.11) in two limits. In the first case we consider a large-field approximation with

$$
\Phi \gg \frac{1}{\sqrt{\gamma}},
$$

and we find that

$$
\begin{aligned}
\frac{d X}{d \Phi} & =\sqrt{\frac{\left(\frac{3 u}{M^{2}} \gamma-1\right)}{2 u \gamma}} \frac{1}{\Phi} \\
\Rightarrow X & =\sqrt{\frac{\left(\frac{3 u}{M^{2}} \gamma-1\right)}{2 u \gamma}} \ln (\sqrt{\gamma} \Phi) .
\end{aligned}
$$

In terms of $\Phi$, we have that

$$
\Phi=\frac{1}{\sqrt{\gamma}} \exp \left(\sqrt{\frac{2 u \gamma}{\left(\frac{3 u}{M^{2}} \gamma-1\right)}} X\right)
$$

The effective potential (6.1) in terms of the canonically normalized field $X$ can be written as

$$
\begin{aligned}
V(X)= & \frac{\mu^{2}}{2 \gamma} \exp \left(\sqrt{\frac{2 u \gamma}{\frac{3 u}{M^{2}} \gamma-1}} 2 X\right) \\
+ & \frac{\lambda\left(1-\frac{1}{\gamma} \exp \left(\sqrt{\frac{2 u \gamma}{\frac{3 u}{M^{2}} \gamma-1}} 2 X\right)\right)^{2}}{4\left(1-\exp \left(\sqrt{\frac{2 u \gamma}{\frac{3 u}{M^{2}} \gamma-1}} 2 X\right)\right)^{2}} .
\end{aligned}
$$

Next, we consider a small-field approximation where

$$
\Phi \ll \frac{1}{\sqrt{\gamma}},
$$

with $\gamma<1$. In this scenario we find that

$$
\frac{d X}{d \Phi}=\frac{1}{\sqrt{2 u}}
$$

which results in

$$
\Phi=\sqrt{2 u} X
$$

Then, the effective potential (6.1) in this case takes the form

$$
V(X)=u \mu^{2} X^{2}+\frac{\lambda\left(1-2 u X^{2}\right)^{2}}{4\left(1-2 \gamma u X^{2}\right)^{2}}
$$

The shape of the potential is shown in Fig. 7 for both cases. In the small-field approximation the factor $\gamma X^{2}$ plays an important role in the predictions of the model. If the contribution of $\gamma$ in the potential (6.19) is very small $\left(\gamma X^{2} \ll 1\right)$, then the tensor-to-scalar ratio is outside the Planck $2 \sigma$ bounds. However, as $\gamma X^{2}$ increases the denominator in Eq. (6.19) becomes important, and some solutions (depending on the other parameters) consistent with the CMB observables appear.

Next, we compute the probability of the inflaton tunneling from the false vacuum at $X_{-}$to the true one located at $X=0$. Following Ref. [63], we analyze the case where the field $X$ interacts with gravity. Then, the Euclidean action of the coupled field $X$ takes the form

$$
S_{E}=\int d^{4} x \sqrt{g}\left(-\frac{1}{2} R+\frac{1}{2} \partial_{\mu} X \partial^{\mu} X+V(X)\right) .
$$

The presence of gravity has important implications for the decay of the false vacuum. Moreover, note also that in the context of general relativity there is an absolute minimum associated with the positive cosmological constant (de Sitter spacetime), in contrast to the field theory case where only potential and energy differences matter.

Denoting the Euclidean action as $S_{E}$, the tunneling probability is defined as a decay rate per unit volume per time by 

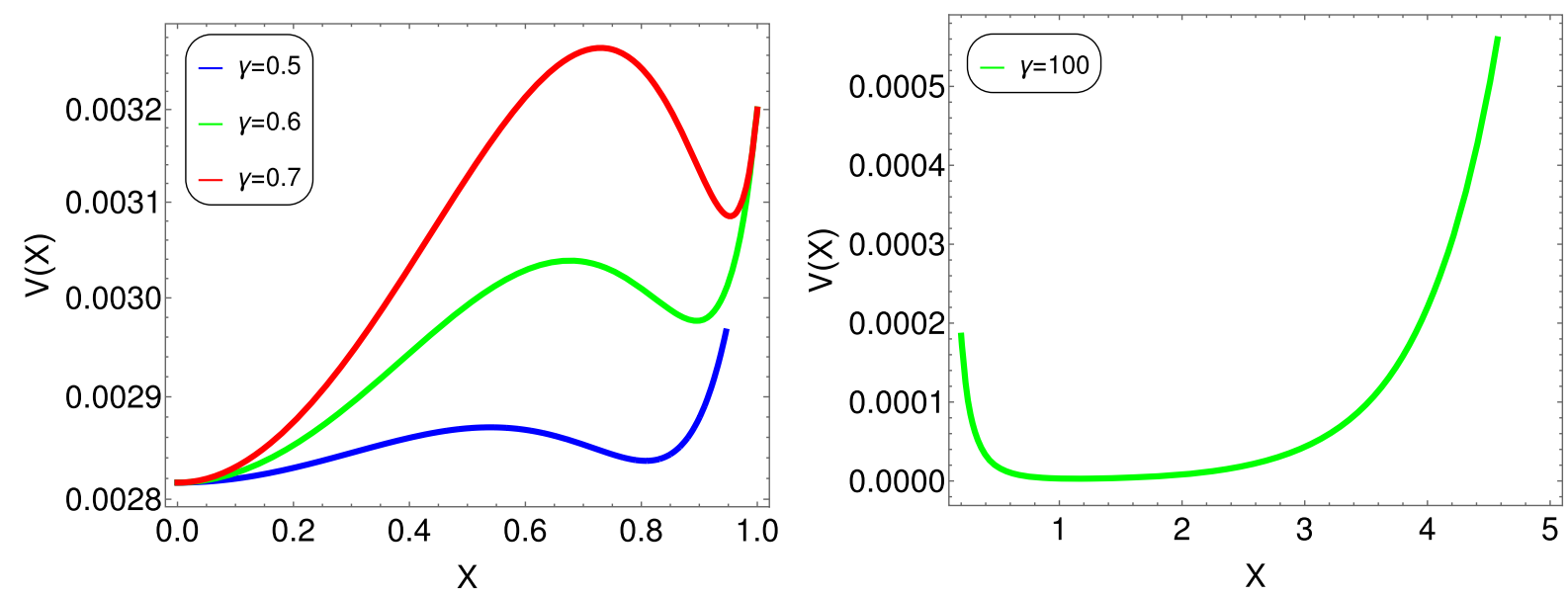

FIG. 7. The left panel shows the shape of the scalar potential for the small-field approximation. In the right panel the shape of the potential in the large-field approximation is presented. Here we use $\lambda / \mu^{2}=1.76, \mu=0.08, M=1$, and $u=1 / 2$.

$$
\Gamma=A e^{-B}, \quad \text { with } \quad B=S_{E}(X)-S_{E}\left(X_{-}\right) .
$$

As in Ref. [63], we look for $O(4)$-symmetric solutions in a Euclidean 4-space that is described by the metric

$$
d s^{2}=d \rho^{2}+\sigma(\rho)^{2}\left(d \Omega_{3}\right)^{2},
$$

where $\left(d \Omega_{3}\right)^{2}$ is the metric of the unit 3 -sphere. The curvature of the 3 -sphere at a given $\rho$ in Eq. (6.22) is given by the scale factor $\sigma(\rho)$. The field equations for the scalar field $X$ and the scale factor $\sigma$ in the time-like case are

$$
\begin{gathered}
X^{\prime \prime}+3 \frac{\sigma^{\prime}}{\sigma} X^{\prime}=\frac{\partial V}{\partial X}, \\
\sigma^{\prime 2}=1+\frac{1}{3} \sigma^{2}\left(\frac{1}{2} X^{\prime 2}-V(X)\right),
\end{gathered}
$$

where the derivatives $X^{\prime}, \sigma^{\prime}$ etc. are taken with respect to the "time" variable $\rho$. For the system of equations (6.23) and (6.24), we adopt the simplest solutions [64], which for $\rho \in\left[0, H_{ \pm}^{-1} \pi\right]$ are given by

$$
X(\rho)=X_{ \pm}, \quad \sigma(\rho)=\frac{1}{H_{ \pm}} \sin \left(H_{ \pm} \rho\right),
$$

where from the field equation $\frac{1}{2} H^{\prime 2}=3 H^{2}-3 V(X)$ at the minima $H_{ \pm}^{\prime}=0$, it turns out that $H_{ \pm}=\sqrt{\frac{V\left(X_{ \pm}\right)}{3}}$. Using the solution (6.25) the action is found to be [64]

$$
S_{E}\left(X_{ \pm}\right)=-2 \pi^{2} \int d \rho \frac{1}{H_{ \pm}^{3}} \sin ^{3}\left(H_{ \pm} \rho\right) V\left(X_{ \pm}\right)=-\frac{24 \pi^{2}}{V\left(X_{ \pm}\right)},
$$

$$
H_{c}^{2}=-\frac{V_{X X}\left(X_{+}\right)}{4}-\frac{\Delta V}{3}
$$

where $V_{X X}=\partial^{2} V / \partial X^{2}$ and, as above, $\Delta V=V\left(X_{+}\right)-$ $V\left(X_{-}\right)$is the height of the barrier. The tunneling coefficient $B$ introduced in Eq. (6.21) is then computed from Eq. (6.26) and reads

$$
B=S_{E}\left(X_{+}\right)-S_{E}\left(X_{-}\right)=-\frac{24 \pi^{2}}{V\left(X_{+}\right)}+\frac{24 \pi^{2}}{V\left(X_{-}\right)} .
$$

In order to estimate the parameter $B$ involved in the decay rate it is sufficient to compute the potential at $X_{ \pm}$. This follows in the next subsection.

\section{A. Numerical results}

The post-inflationary Universe is described by radiation, matter, and vacuum energy density. Furthermore, it is assumed that the bubble nucleation rate $\Gamma$ in the past satisfies $\Gamma \geq \Gamma_{0}$, where $\Gamma_{0}$ is its current Minkowski-space value. The constraint on the nucleation rate $\Gamma_{0}$ from the post-inflationary era was given in Ref. [65], which further implies the bound $B_{0} \gtrsim 540$. Before presenting the numerical details, we first mention the scanning ranges. The shape of the potential and the existence of false minima depend on two parameters: the ratio $\lambda / \mu^{2}$ and $\gamma$. From the previous analytical calculation we already found that false minima occur within the following bounds:

$$
1<\frac{\lambda}{\mu^{2}} \leq \frac{27}{16}, \quad \frac{\left(1-\sqrt{\frac{11}{27}}\right)}{2} \leq \gamma<1
$$

In the following the value of $\mu$ will be fixed to $\mu=\frac{1}{2}$. Hence, we perform the numerical scan varying $\lambda / \mu^{2}$ and $\gamma$ according to Eq. (6.29).

There are two types of solutions that contribute to vacuum decay in de Sitter space: the CdL solution [63] which crosses the barrier through tunneling, and the Hawking-Moss solution (HM) [66] where the inflaton is 

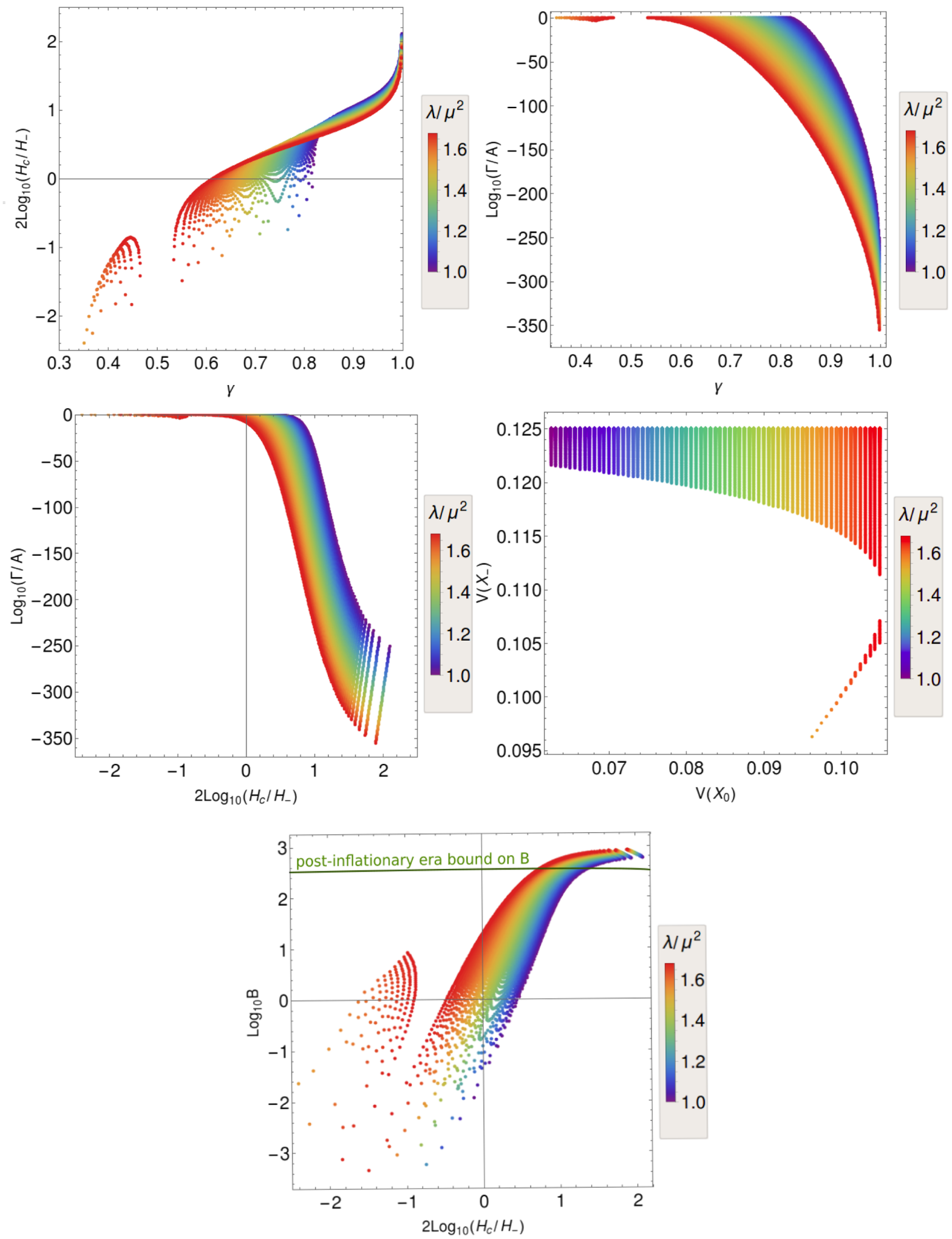

FIG. 8. Different results for the probability that the inflaton tunnels from the false vacuum at $X_{-}$to the true vacuum $X=0$. Here we use $\mu=0.5$. 
on the top of the barrier (for a more detailed discussion, see Ref. [64]). In Eq. (6.25) we defined $H_{-}$which is actually the background Hubble rate in the false vacuum. The condition for HM solutions to contribute to vacuum decay is $H \rightarrow H_{c}$, where $H_{c}$ is the critical Hubble rate defined in Eq. (6.27). The second term of the critical Hubble rate generally contributes significantly if the height difference between the top of the barrier and the false vacuum is comparable to the Planck mass.

In Fig. 8, the top-left plot shows $\gamma$ vs the ratio of the Hubble scale and the top-right plot shows $\gamma$ vs the decay width, where the rainbow color bar shows how these solutions vary with the ratio of $\lambda / \mu^{2}$. We see from the top-left plot that for a low Hubble rate we have solutions corresponding to the HM instanton which contribute to vacuum decay. Recall that the HM instanton solution is the one for which the inflaton stays at the top of the barrier $X(\rho)=X_{+}$. This describes the situation where the inflaton climbs up the potential barrier instead of tunneling. On the other hand, when the Hubble rate increases, i.e., $H_{-}<H_{c}$, standard CdL solutions appear. In the top-left panel of Fig. 8, the solutions below the horizontal black line correspond to HM. On the other hand, those solutions above the black horizontal line correspond to the CdL case. The middle-left panel of Fig. 8 shows the plot between the ratio of Hubble scales and the decay rate, while in the middle-right panel one can see the values of the potential at both minima. In these scans we only show plots with solutions in which $V\left(X_{-}\right)>V\left(X_{0}\right)$, so the motion of the inflaton field is from right to left. The lower panel shows the plot between the ratio of Hubble scales and the parameter $B$, where the horizontal line in the figure shows the bound on $B$ coming from the post-inflationary era. Based on the numerical value of $B$, we can categorize our false vacuum into stable, metastable, and unstable. From the plot we see that $B>B_{0}$ (where $B_{0}=540$ ) is a region where $\Gamma<\Gamma_{0}$ and we can infer that the false vacuum is stable. Similarly, $B \simeq B_{0}$ corresponds to metastable and $B<B_{0}$ represents the unstable region.

\section{CONCLUSION}

In this work we investigated various cosmological implications of a generic model based on the extension of the SM gauge symmetry by a $U(1)_{B-L}$ factor, with special emphasis on the issues of inflation, leptogenesis, and baryogenesis as well as the physics of the gravitino. The model can be naturally embedded in a unified gauge group with a symmetry-breaking scale around $M_{\mathrm{GUT}}=2 \times 10^{16} \mathrm{GeV}$. The spectrum of the model consists of the MSSM content extended by a neutral singlet field $S$, and a pair of Higgs MSSM singlets $(H, \bar{H})$ carrying opposite charge under $U(1)_{B-L}$. An appropriate $R$ symmetry prevents all of the dangerous terms in the superpotential, whereas a suitable fourth-order nonrenormalizable term providing Majorana mass to the right-handed neutrino is left intact to realize the seesaw mechanism. The most general effective potential consists of the $F$ and $D$ parts as well and contributions coming from soft supersymmetry-breaking terms. The $F$ part arises from the Kähler function, assuming standard noscale supergravity, and the $D$ terms contain the usual contributions associated with the gauge sector. Under mild constraints on the parameters involved in the scalar potential, it is readily realized that the inflationary scenario is naturally implemented. The cosmological observables, including the tensor-to-scalar ratio $r$, the spectral index $n_{s}$, etc., were computed and discussed in detail for various limiting cases of the effective potential. In the present analysis, a wide viewpoint was taken to encompass future perspectives on the possible determination of the supersymmetry-breaking scale $m_{\text {SUSY }}$. Thus, in this context, by varying $m_{\mathrm{SUSY}}$ from a few $\mathrm{TeV}$ up to $10^{6} \mathrm{TeV}$, while fixing the spectral index to its central value $n_{s}=0.9655$, we found that the tensor-to-scalar ratio lies in the range $r \in\left[10^{-2}-10^{-3}\right]$ which is consistent with the latest Planck data.

We examined in detail the physics related to the nature of the gravitino and considered possible scenarios, including that it is the LSP. Among other possibilities, we found that a stable LSP gravitino is easily accommodated in our setup, and as such it can be considered as a DM candidate. If the gravitino is not the LSP, we found that a short-lived gravitino is always viable as long as its mass is $m_{3 / 2}>25 \mathrm{TeV}$, whereas the mass of a long-lived one should lie in the region $10<m_{3 / 2}<25 \mathrm{TeV}$.

For high SUSY scales the reheating temperature $T_{r}$ is bounded by $\Omega_{\mathrm{LSP}}$, whereas for a TeV-scale SUSY case the reheating temperature is bounded by BBN predictions. Furthermore, the model predicts an inflaton mass around $10^{12} \mathrm{GeV}$. On the other hand, the variation of the righthanded heavy Majorana scale depends on $T_{r}$ and ranges between $M_{N} \sim 10^{8}$ and $10^{12} \mathrm{GeV}$. Values close to the upper scale are sufficient to realize the seesaw mechanism and obtain light neutrino masses consistent with the oscillation experiments. Finally, we identified regions of the parameter space where the potential in addition to the true vacuum it also displays a false minimum. For this particular case, we discussed quantum tunneling effects with the inflaton field penetrating the barrier, and computed its decay width to the true vacuum using standard techniques developed by Coleman and De Luccia [63].

\section{ACKNOWLEDGMENTS}

The authors are thankful to Mansoor Ur Rehman and Fariha K. Vardag for fruitful discussions. The work of G. K. L. was supported by the "Hellenic Foundation for Research and Innovation (H. F. R. I.) under the "First Call for H. F. R. I. Research Projects to support Faculty members and Researchers and the procurement of high-cost research equipment grant" (Project No. 2251). 
[1] A. A. Starobinsky, Phys. Lett. 91B, 99 (1980); V. F. Mukhanov and G. V. Chibisov, Pis'ma Zh. Eksp. Teor. Fiz. 33, 549 (1981) [JETP Lett. 33, 532 (1981)].

[2] A. Guth, Phys. Rev. D 23, 347 (1981).

[3] A. Albrecht and P. J. Steinhardt, Phys. Rev. Lett. 48, 1220 (1982).

[4] P. A. R. Ade et al. (BICEP2 and Keck Array Collaborations), Phys. Rev. Lett. 121, 221301 (2018).

[5] Y. Akrami et al. (Planck Collaboration), Astron. Astrophys. 641, A10 (2020).

[6] J. Ellis, D. V. Nanopoulos, and K. A. Olive, Phys. Rev. Lett. 111, 111301 (2013); 111, 129902(E) (2013).

[7] E. Cremmer, S. Ferrara, C. Kounnas, and D. V. Nanopoulos, Phys. Lett. 133B, 61 (1983).

[8] J. R. Ellis, C. Kounnas, and D. V. Nanopoulos, Nucl. Phys. B247, 373 (1984).

[9] A. B. Lahanas and D. V. Nanopoulos, Phys. Rep. 145, 1 (1987).

[10] M. B. Einhorn and D. R. T. Jones, J. High Energy Phys. 03 (2010) 026.

[11] S. Ferrara, R. Kallosh, A. Linde, A. Marrani, and A. Van Proeyen, Phys. Rev. D 83, 025008 (2011).

[12] S. Ferrara, R. Kallosh, A. Linde, A. Marrani, and A. Van Proeyen, Phys. Rev. D 82, 045003 (2010).

[13] J. Ellis, M. A. G. Garcia, N. Nagata, D. V. Nanopoulos, K. A. Olive, and S. Verner, Int. J. Mod. Phys. D 29, 2030011 (2020).

[14] C. Pallis and N. Toumbas, J. Cosmol. Astropart. Phys. 12 (2011) 002.

[15] J. Ellis, H. J. He, and Z. Z. Xianyu, Phys. Rev. D 91, 021302 (2015).

[16] J. Ellis, H. J. He, and Z. Z. Xianyu, J. Cosmol. Astropart. Phys. 08 (2016) 068.

[17] W. Ahmed and A. Karozas, Phys. Rev. D 98, 023538 (2018).

[18] G. K. Leontaris, N. Okada, and Q. Shafi, Phys. Lett. B 765, 256 (2017); M. K. Parida, B. P. Nayak, R. Satpathy, and R. L. Awasthi, J. High Energy Phys. 04 (2017) 075; A. Karozas, G. K. Leontaris, Q. Shafi, and I. Tavellaris (to be published).

[19] H. M. Lee, J. Cosmol. Astropart. Phys. 08 (2010) 003.

[20] F. L. Bezrukov, A. Magnin, and M. Shaposhnikov, Phys. Lett. B 675, 88 (2009); A. De Simone, M. P. Hertzberg, and F. Wilczek, Phys. Lett. B 678, 1 (2009); A. O. Barvinsky, A. Y. Kamenshchik, C. Kiefer, A. A. Starobinsky, and C. Steinwachs, J. Cosmol. Astropart. Phys. 12 (2009) 003; N. Okada, M. U. Rehman, and Q. Shafi, arXiv:0911.5073.

[21] N. Okada, M. U. Rehman, and Q. Shafi, Phys. Rev. D 82, 043502 (2010); A. Linde, M. Noorbala, and A. Westphal, J. Cosmol. Astropart. Phys. 03 (2011) 013; N. Okada, M. U. Rehman, and Q. Shafi, Phys. Lett. B 701, 520 (2011); C. Pallis and Q. Shafi, J. Cosmol. Astropart. Phys. 03 (2015) 023; N. Bostan, Ö. Güleryüz, and V. N. Şenoğuz, J. Cosmol. Astropart. Phys. 05 (2018) 046; N. Bostan and V. N. Senoguz, arXiv:1907.06215.

[22] A. R. Liddle and D. H. Lyth, Cosmological inflation and large scale structure.

[23] A. D. Linde, Phys. Rev. D 49, 748 (1994).

[24] G. R. Dvali, Q. Shafi, and R. K. Schaefer, Phys. Rev. Lett. 73, 1886 (1994).
[25] E. J. Copeland, A. R. Liddle, D. H. Lyth, E. D. Stewart, and D. Wands, Phys. Rev. D 49, 6410 (1994).

[26] A. D. Linde and A. Riotto, Phys. Rev. D 56, R1841 (1997); V. N. Senoguz and Q. Shafi, Phys. Rev. D 71, 043514 (2005); M. U. Rehman, Q. Shafi, and J. R. Wickman, Phys. Lett. B 683, 191 (2010).

[27] R. Jeannerot, S. Khalil, G. Lazarides, and Q. Shafi, J. High Energy Phys. 10 (2000) 012.

[28] G. Lazarides and C. Panagiotakopoulos, Phys. Rev. D 52, R559 (1995).

[29] N. Arkani-Hamed and S. Dimopoulos, J. High Energy Phys. 06 (2005) 073.

[30] G. F. Giudice and A. Romanino, Nucl. Phys. B699, 65 (2004); B706, 487(E) (2005).

[31] W. Buchmuller, V. Domcke, and K. Schmitz, Nucl. Phys. B862, 587 (2012).

[32] W. Buchmuller, V. Domcke, K. Kamada, and K. Schmitz, arXiv:1309.7788.

[33] W. Ahmed, S. Raza, Q. Shafi, C. S. Un, and B. Zhu, J. High Energy Phys. 01 (2021) 161.

[34] G. R. Dvali, G. Lazarides, and Q. Shafi, Phys. Lett. B 424, 259 (1998).

[35] S. F. King and Q. Shafi, Phys. Lett. B 422, 135 (1998).

[36] R. Deen, B. A. Ovrut, and A. Purves, Phys. Lett. B 762, 441 (2016).

[37] G. F. Giudice and A. Masiero, Phys. Lett. B 206, 480 (1988).

[38] W. Buchmuller, L. Covi, and D. Delepine, Phys. Lett. B 491, 183 (2000).

[39] W. Buchmüller, V. Domcke, K. Kamada, and K. Schmitz, J. Cosmol. Astropart. Phys. 07 (2014) 054.

[40] W. Buchmuller, V. Domcke, H. Murayama, and K. Schmitz, Phys. Lett. B 809, 135764 (2020).

[41] C. Pallis, Universe 4, 13 (2018); Eur. Phys. J. C 78, 523 (2018).

[42] M. Y. Khlopov and A. D. Linde, Phys. Lett. 138B, 265 (1984).

[43] J. R. Ellis, J. E. Kim, and D. V. Nanopoulos, Phys. Lett. 145B, 181 (1984).

[44] M. Kawasaki and T. Moroi, Prog. Theor. Phys. 93, 879 (1995).

[45] R. H. Cyburt, J. R. Ellis, B. D. Fields, and K. A. Olive, Phys. Rev. D 67, 103521 (2003).

[46] M. Fujii, M. Ibe, and T. Yanagida, Phys. Lett. B 579, 6 (2004).

[47] T. Gherghetta, G. F. Giudice, and A. Riotto, Phys. Lett. B 446, 28 (1999).

[48] T. Gherghetta, G. F. Giudice, and J. D. Wells, Nucl. Phys. B559, 27 (1999).

[49] P. Andre et al. (PRISM Collaboration), arXiv:1306.2259.

[50] T. Matsumura et al., J. Low Temp. Phys. 176, 733 (2014).

[51] A. Kogut et al., J. Cosmol. Astropart. Phys. 07 (2011) 025.

[52] F. Finelli et al. (CORE Collaboration), J. Cosmol. Astropart. Phys. 04 (2018) 016.

[53] G. Lazarides, M. U. Rehman, Q. Shafi, and F. K. Vardag, Phys. Rev. D 103, 035033 (2021).

[54] N. Okada and Q. Shafi, Phys. Lett. B 775, 348 (2017).

[55] M. Bolz, A. Brandenburg, and W. Buchmuller, Nucl. Phys. B606, 518 (2001).

[56] J. Pradler and F. D. Steffen, Phys. Rev. D 75, 023509 (2007). 
[57] V. S. Rychkov and A. Strumia, Phys. Rev. D 75, 075011 (2007).

[58] H. Eberl, I. D. Gialamas, and V. C. Spanos, Phys. Rev. D 103, 075025 (2021).

[59] T. A. Vami (ATLAS and CMS Collaborations), Proc. Sci., LHCP2019 (2019) 168 [arXiv:1909.11753].

[60] M. Aaboud et al. (ATLAS Collaboration), Phys. Rev. D 97, 112001 (2018).

[61] M. Kawasaki, K. Kohri, and T. Moroi, Phys. Rev. D 71, 083502 (2005); M. Kawasaki, K. Kohri, T. Moroi, and A. Yotsuyanagi, Phys. Rev. D 78, 065011 (2008);
M. Kawasaki, K. Kohri, T. Moroi, and Y. Takaesu, Phys. Rev. D 97, 023502 (2018).

[62] D. Hooper and T. Plehn, Phys. Lett. B 562, 18 (2003).

[63] S. R. Coleman and F. De Luccia, Phys. Rev. D 21, 3305 (1980).

[64] I. Antoniadis, O. Lacombe, and G. K. Leontaris, Eur. Phys. J. C 80, 1014 (2020).

[65] T. Markkanen, A. Rajantie, and S. Stopyra, Front. Astron. Space Sci. 5, 40 (2018).

[66] S. W. Hawking and I. G. Moss, Adv. Ser. Astrophys. Cosmol. 3, 154 (1987). 